\title{
Size, shape, orientation, speed, and duration of GPS equatorial anomaly scintillations
}

\author{
P. M. Kintner and B. M. Ledvina \\ School of Electrical and Computer Engineering, Cornell University, Ithaca, New York, USA \\ E. R. de Paula and I. J. Kantor \\ Aeronomy Division, DAE/CEA, Instituto Nacional de Pesquisas Espaciai, São José dos Campos, São Paulo, Brazil
}

Received 29 January 2003; revised 21 August 2003; accepted 5 February 2004; published 7 April 2004.

[1] GPS L1 C/A signal scintillation data were collected at the equatorial anomaly over a period of three months using five receivers spaced on magnetic east-west and north-south axes to examine the speed, orientation, shape, width, and duration of GPS scintillation fade patterns. The nighttime speeds were primarily eastward in the range of $100-200 \mathrm{~m} / \mathrm{s}$ with a significant spread to both larger values and negative (westward) values as expected, given the known behavior of ionospheric drifts and GPS signal path movement. The characteristic velocity was found to be small so that the true velocity was equal to the apparent velocity to a very good approximation. The orientation of the scintillation fade patterns was organized by a simple projection model of the magnetic field along the GPS signal path onto the horizontal plane when the signal paths were aligned no closer than $60^{\circ}$ from the magnetic field. The shape of the scintillation fade pattern was greatly elongated in the magnetic north-south direction, and no change could be detected over a distance of $1 \mathrm{~km}$. The east-west widths of the scintillation fade patterns were variable, but after normalizing to the elevation angle, accounting for the fade orientation, and eliminating signal paths within $60^{\circ}$ of the magnetic field, an organized scale length of about $450 \mathrm{~m}$ was determined. The duration of the scintillation fade patterns was examined using the optimal cross-correlation amplitude as a measure of change. For a $5 \mathrm{~s}$ duration, $49 \%$ of the optimal cross-correlation amplitudes exceed a value of 0.8 . INDEX TERMS: 2439 Ionosphere: Ionospheric irregularities; 6934 Radio Science: Ionospheric propagation (2487); 6979 Radio Science: Space and satellite communication; KEYWORDS: ionospheric propagation, ionospheric irregularities, space and satellite communication

Citation: Kintner, P. M., B. M. Ledvina, E. R. de Paula, and I. J. Kantor (2004), Size, shape, orientation, speed, and duration of GPS equatorial anomaly scintillations, Radio Sci., 39, RS2012, doi:10.1029/2003RS002878.

\section{Introduction}

[2] The propagation of electromagnetic waves through turbulent media produces scintillations through diffraction. Frequently, turbulent media occur naturally, such as in the interstellar medium, the solar wind, and the ionosphere [Yeh et al., 1975; Yeh and Liu, 1982; Tokumaru et al., 2000; Rickett, 2001; Vats et al., 2001]. In these cases, investigating scintillations yields information about the process of diffraction in random media, the bulk properties of the media in which the turbulence is embedded (such as flow), and the nature

Copyright 2004 by the American Geophysical Union. 0048-6604/04/2003RS002878\$11.00 of the turbulence producing the diffraction. These investigations have led to new discoveries and are a powerful technique for probing distant cosmic plasmas. Conversely, in some examples, scintillations are less welcome. They degrade signal amplitude, introduce phase fluctuations, and, in the case of trans-ionospheric propagation of satellite signals, can make the links unusable. Hence understanding the physical nature of scintillations is important for engineers and technologists as well as for scientists. As the use of communication and navigation signals originating from satellites becomes increasingly embedded in our technological fabric, including safety-of-life applications, a clear understanding and description of ionospheric scintillations becomes essential. 
[3] Typically, the study of ionospheric scintillations has focused on reduced data sets or indirect measures of scintillation activity. For example, the $\mathrm{S} 4$ index is a measure of the signal amplitude RMS fluctuations compared to the mean value of the signal amplitude. An indirect approach such as ROTI (root mean square of deviation of $d \mathrm{TEC} / d t$ ) examines changes in TEC values to infer the presence of scintillations [Aarons et al., 1997; Musman et al., 1997; Pi et al., 1997; Beach and Kintner, 1999]. These methods are particularly useful for understanding the climatology of ionospheric scintillations and answering the questions of when, where, and how much [Aarons, 1982; Aarons et al., 1983; Aarons, 1993; Doherty et al., 2000]. These measures are also useful for nowcasting, prediction, or the validation of ionospheric simulations and models. However, the analysis of reduced data sets does not address the questions of size, shape, orientation, speed, and duration of the fading scintillations patterns.

[4] GPS signals are affected by ionospheric scintillations. GPS receivers operate by tracking the signal code and/or carrier phase with a tracking loop to detect time of arrival. Several different kinds of tracking loops are used, depending on the application and design choices but all of them are sensitive at some level to reduced signal amplitude and the duration of the reduced signal amplitude. How a GPS receiver responds to ionospheric scintillations depends partly on the receiver design and partly on the signal fade characteristics in the receiver reference frame, which may be moving. Earlier, Kintner et al. [2001] demonstrated that the duration of a scintillation signal fade will depend on the motion of a GPS receiver, since signal fades are produced by a spatial pattern of amplitude fluctuations translating across the ground at a speed given, in part, by the ionospheric flow and, in part, by the motion of the signal puncture point in the ionosphere. This paper is partially an extension of the Kintner et al. [2001] paper that further examines the properties of signal fades and the conditions leading to velocity matching or resonance. These observations are important in understanding how airborne GPS receivers will respond in a scintillating environment. Additionally, it is of interest to scientists designing and investigating scintillation experiments, to GPS systems designers who need to understand the detailed properties of scintillations, and to scientists investigating the ionosphere and plasma irregularities.

[5] This paper is organized by first briefly reviewing the study of ionospheric scintillations and then describing an experiment to investigate the properties of GPS L1 signal fades at the equatorial anomaly. This experiment led to a large data set collected over a period of three months using multiple receivers with spatial separations of several hundred meters to nearly $1 \mathrm{~km}$. From this data set the rapidly sampled amplitudes are reduced to cross-correlation and autocorrelation functions and parameters describing those functions. Using these parameters, we then investigate the speed, orientation and shape, size, and duration of GPS L1 signal fades in the C/A code. In reading this paper, it is important to realize that the temporal fluctuations in signal amplitude, produced by diffraction in a turbulent ionosphere and experienced by a stationary receiver, are the result of a spatial pattern translating across the ground and evolving more slowly in its own reference frame. We will refer to both the temporal fluctuations and the spatial pattern as scintillations.

\section{Overview of Ionospheric Scintillations}

[6] Scintillations are typically produced in a turbulent medium where small changes in the index of refraction produce scattering. For the case of radio waves in the earth's ionosphere, whose frequencies are much larger than the electron gyrofrequency $(1-1.5 \mathrm{MHz})$ and the electron plasma frequency, the index of refraction becomes

$$
n=\sqrt{1-\frac{\omega_{p e}^{2}}{\omega^{2}}} \cong 1-\frac{1}{2} \frac{\omega_{p e}^{2}}{\omega^{2}}=1-\frac{2 \pi^{2} e^{2}}{m e} \frac{n_{e}}{\omega^{2}},
$$

where $\omega_{p e}$ is the electron plasma frequency $\left(\sqrt{4 \pi n_{e} e^{2} / m_{e}}\right)$, $\omega$ is the radio wave angular frequency, and $n_{e}$ is the ionospheric electron density. Since $\omega_{p e}^{2} \propto n_{e}$, the most significant scintillations will occur where the ionospheric electron density is the largest if the ionosphere is turbulent. The ionosphere is most dense at the equatorial or Appleton anomalies where diurnal tides in the thermosphere force the equatorial plasma upward during the day. This plasma then flows down magnetic field lines to regions roughly $\pm 15^{\circ}$ from the magnetic equator where the electron density may reach values of a few $10^{6} \mathrm{~cm}^{-3}$. These regions of enhanced density form during the day and last through the evening hours or longer, depending on the altitude and associated recombination time. The anomalies also show an 11-year, solar-cycle dependence since the ionosphere is produced by solar EUV radiation, which is more intense and variable during solar sunspot maximum.

[7] After sunset, at the magnetic equator the $F$ region ionosphere, where most of the ionospheric electron density is contained, frequently becomes turbulent and produces absolute levels of electron density fluctuations exceeding those typically found anywhere else in the ionosphere. This phenomenon is called equatorial spread $F$ and has been studied extensively for more than 5 decades. The morphology of equatorial spread $F$ has been well documented, including its seasonal dependence and its day-to-day unpredictability [see, e.g., Farley et al., 1970; Kelley et al., 1976; Woodman and 
La Hoz, 1976; McClure et al., 1977; Fejer et al., 1981, 1999]. The spectral range of density fluctuations within equatorial spread $F$ has also been well documented through remote sensing and in situ measurements, which show a range from hundreds of $\mathrm{km}$ to $\mathrm{cm}$. For the most part, the irregularities are carried as a passive scalar in the ionospheric reference frame, which is primarily eastward during darkness but with substantial vertical components during the early evening [Hysell, 1999].

[8] The general picture of equatorial scintillations has been well established at many levels: statistical comparisons, single-event studies, and in situ measurements [Basu et al., 1977, 1980; Basu and Basu, 1981; DasGupta et al., 1983; Aarons et al., 1996; Aarons, 1997a, 1997b; Abdu et al., 1998; Beach and Kintner, 1999]. These studies conclude that transionospheric radio wave scintillations are most significant within the equatorial anomalies and most directly associated with equatorial spread $F$. At L-band frequencies the scintillation fade amplitudes can exceed $20 \mathrm{~dB}$. Aarons et al. [1983] and Basu et al. [1986] first realized that equatorial irregularities, particularly those associated with the anomalies, could produce fading at $\mathrm{GHz}$ frequencies and potentially affect GPS signals. This realization was confirmed with simultaneous radar and GPS measurements of TEC [Kelley et al., 1996]. Many examples of GPS receivers being used to observe the disturbed equatorial ionosphere and simultaneously having their performance degraded now exist, both on the ground [Bishop et al., 1994; Kintner et al., 2001] and in space [Goodman and Kramer, 2001; Kramer and Goodman, 2001; P. R. Strauss et al., GPS occultation sensor observations of ionospheric scintillation, submitted to Geophysical Research Letters, 2002].

[9] One feature of equatorial spread $F$ scintillations was discovered by observing fades from an airplane at VHF frequencies [Aarons et al., 1980]. Investigators observed that occasionally the time span of individual fades was greatly extended and concluded that the airborne receiver was matching the velocity of the drifting scintillation fade pattern. Kintner et al. [2001] demonstrated that this was also a feature of GPS signals in which GPS signal puncture-point velocities sometimes could balance the ionospheric drift velocity to create lengthened fades in ground-based, stationary receivers and potentially in airborne receivers. In fact, it is more likely to occur in airborne receivers where velocity matching conditions are less restrictive. Given the potential to resonate with moving fades and the potential for GPS receiver tracking to fail in the presence of deep and lengthy fades, a description of fading properties in the spatial domain and the drifting reference frame is required. The description is required, for example, to understand how resonance will occur when the fade drift velocity is primarily east-west, while airborne receivers

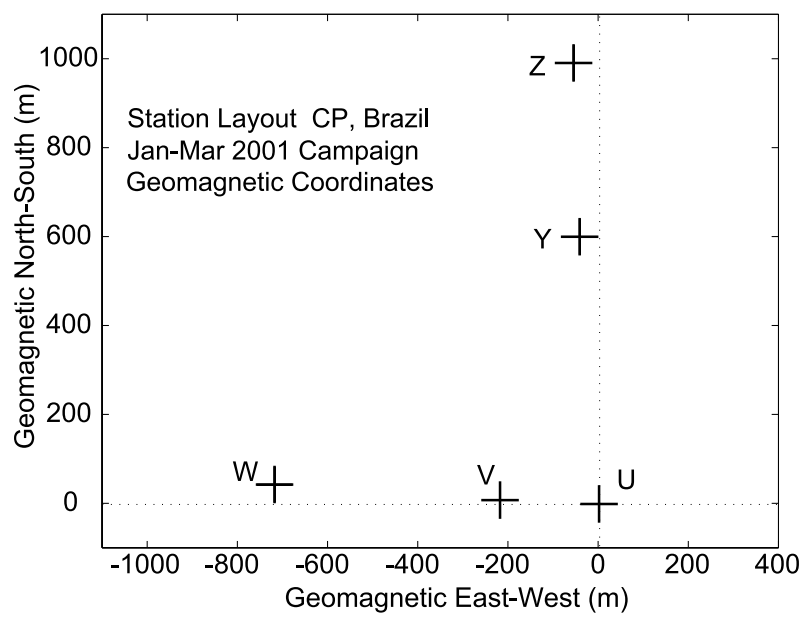

Figure 1. Schematic layout of the five Cornell scintillation receivers labeled $\mathrm{U}, \mathrm{V}, \mathrm{W}, \mathrm{Y}$, and $\mathrm{Z}$.

typically have both east-west and north-south velocity components. In this paper we provide the missing description to complement decades of work in characterizing scintillations as a temporal phenomenon in a static reference frame.

\section{Experimental Design}

[10] To investigate the size, shape, speed, and duration of ionospheric scintillation fade patterns we chose the location of Cachoeira Paulista, Brazil because it is located under the equatorial anomaly at a magnetic latitude and longitude of about $-17^{\circ}$ and $22^{\circ}$, respectively, and because the Instituto Nacional de Pesquisas Espaciais (INPE) operates a large facility capable of running our receivers. The campaign was conducted from January through March, 2001. Five Cornell scintillation receivers (SCINTMON) based on the Plessey chip set [Beach and Kintner, 2001] were used to record GPS L1 C/A amplitude data at 50 samples per second. These receivers record the wideband signal amplitude on 11 channels and compare it with the noise level measured on a 12th channel (which is not tracking) to yield signalto-noise ratios for up to 11 satellites. This receiver has been used extensively in the South American sector, and a Linux-based version displaying real time S4 data from several stations can be viewed at the following web site: http://gps.ece.cornell.edu.

[11] Figure 1 shows the five receiver locations in a roughly right-angle configuration. The receivers are labeled U, V, W, Y and Z. Receivers W and V are located in the magnetic west direction from $U$ with a maximum westward separation of $715 \mathrm{~m}$ while receivers $\mathrm{Y}$ and $\mathrm{Z}$ are located in the magnetic northward direction with a 
Table 1. Coordinates of the Four SCINTMON Receivers Relative to the U Receiver

\begin{tabular}{cccc}
\hline Receiver & $x$ Coordinate, $\mathrm{m}$ & $y$ Coordinate, $\mathrm{m}$ & Altitude, $\mathrm{m}$ \\
\hline $\mathrm{V}$ & -218 & 8.6 & 2.3 \\
$\mathrm{~W}$ & -715 & 40.5 & 24.8 \\
$\mathrm{Y}$ & -39.6 & 600 & 13.4 \\
$\mathrm{Z}$ & -54.7 & 991 & 11.5 \\
\hline
\end{tabular}

maximum separation of 991 meters. The coordinates of the five receivers are shown in Table 1 using magnetic coordinates, assigning the $\mathrm{U}$ receiver to the origin, and letting west be in the $-\mathrm{x}$ direction while north is in the $+y$ direction. The geographic coordinates of the $\mathrm{U}$ receiver were a latitude of $-22.688601^{\circ}$ and a longitude of $-44.999929^{\circ}$.

[12] The relatively large separations for the receivers were chosen because previous shorter separations were not able to resolve the characteristic velocities of the scintillation fade patterns [Briggs et al., 1950; Kil et al., 2000] and because we wished to investigate the north-south extent of the scintillation fade patterns. This latter point will take on special significance when we consider the fade orientation. In addition, all antennas used a ground plane and were more than $100 \mathrm{~m}$ from any reflecting object, producing a very low multipath environment.

[13] The five receivers were operated each night as continuously as possible for the three months of the campaign. In the data analyzed below we will make use of receiver set $\mathrm{U}, \mathrm{V}$, and $\mathrm{W}$ and the receiver set $\mathrm{U}, \mathrm{W}$, and $\mathrm{Z}$. For the receiver set $\mathrm{U}, \mathrm{V}$, and $\mathrm{W}$, data were collected on 45 nights. For the receiver set $\mathrm{U}, \mathrm{W}$, and $\mathrm{Z}$, data were also collected on 45 nights with about $80 \%$ overlap with the $\mathrm{U}, \mathrm{V}$, and $\mathrm{W}$ data. This yielded a very large data set of about 40 Gbytes.

[14] This large data set was reduced in size by creating normalized autocorrelation and cross-correlation functions and by recording key parameters from these correlation functions. Figure 2 shows an example of cross-correlation functions among some of the receiver pairs for a single satellite signal. The autocorrelation and cross-correlation functions were constructed from $40 \mathrm{~s}$ of data sampled at 50 samples per second. For each correlation function we recorded the correlation peak value (just 1 for the autocorrelation function), the correlation function width at the $3 \mathrm{~dB}$ point $(0.5$ of the peak value), the lag of an associated cross-correlation function yielding the maximum (or optimum) cross-correlation value, and the times at which the autocorrelation function has the same value as the peak value of the crosscorrelation function. This last point will be significant when we consider the characteristic velocity of the scintillation fade patterns in section 4 . In general, we selected data when $\mathrm{S} 4>0.5$ and the elevation angles of the satellites were greater than $10^{\circ}$. By only reflecting data above the large S4 floor, virtually all multipath effects were eliminated. In addition, if the peak value of the cross-correlation function was less than 0.3 we rejected the data because of the possibility of identifying a false peak. This process of selection yielded 10,214 simultaneous correlations over 45 days for each of the U, $\mathrm{V}$, and $\mathrm{W}$ receivers, and 9097 simultaneous correlations over 45 days for each of the $\mathrm{U}, \mathrm{W}$, and $\mathrm{Z}$ receivers.

[15] The next step was to validate the analysis technique by making a simple comparison of velocity through examining cross-correlation functions between the U-W, U-V, and V-W receiver pairs. Figure 3 shows the velocity $(\mathrm{m} / \mathrm{s})$ estimated with the receiver pairs $\mathrm{U}-\mathrm{V}$ compared to the velocity estimated with the receiver pairs U-W in the upper panel, and in the lower panel, the velocity estimated with the receiver pairs V-W compared to the velocity estimated with the receiver pairs U-W. These velocities represent the true velocity as opposed to the apparent velocity, which is discussed in section 4. Under the equatorial anomalies, ionospheric velocities are primarily east-west with values of up to a few hundred meters per second [Kil et al., 2000]. To obtain the scintillation fade pattern velocity, the GPS signal puncture-point velocity must also be included, which ranges from $-100 \mathrm{~m} / \mathrm{s}$ to $300 \mathrm{~m} / \mathrm{s}$ [Kintner et al., 2001]. The large velocity values (greater than $500 \mathrm{~m} / \mathrm{s}$ ) seen in Figure 3 are apparently real but not as common as the figure suggests. The large velocities may result from

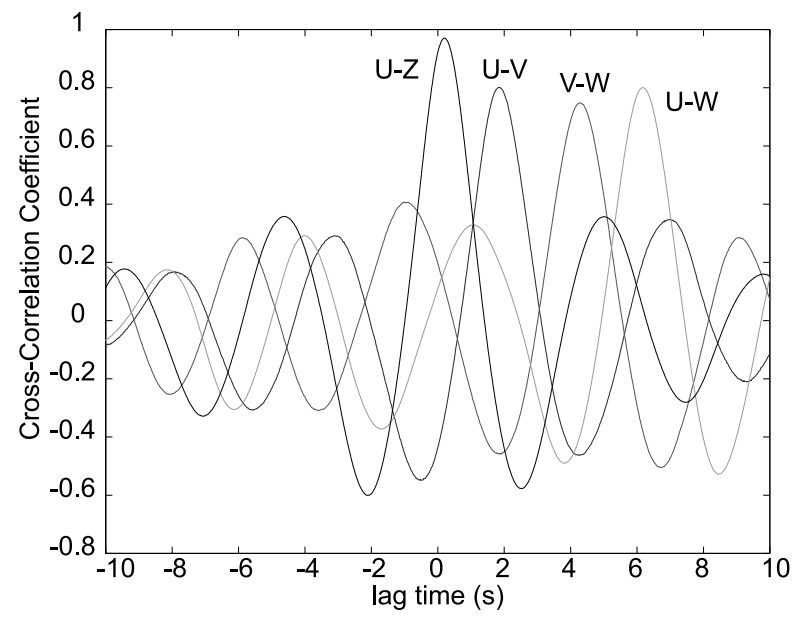

Figure 2. Examples of normalized cross-correlation functions between different receiver pairs for a single GPS satellite signal. Note that the receiver pair aligned northsouth (U-Z) has larger optimal correlation values and smaller times of optimal correlation compared to the receivers in the east-west direction (U-V, V-W, and U-W). See color version of this figure at back of this issue. 

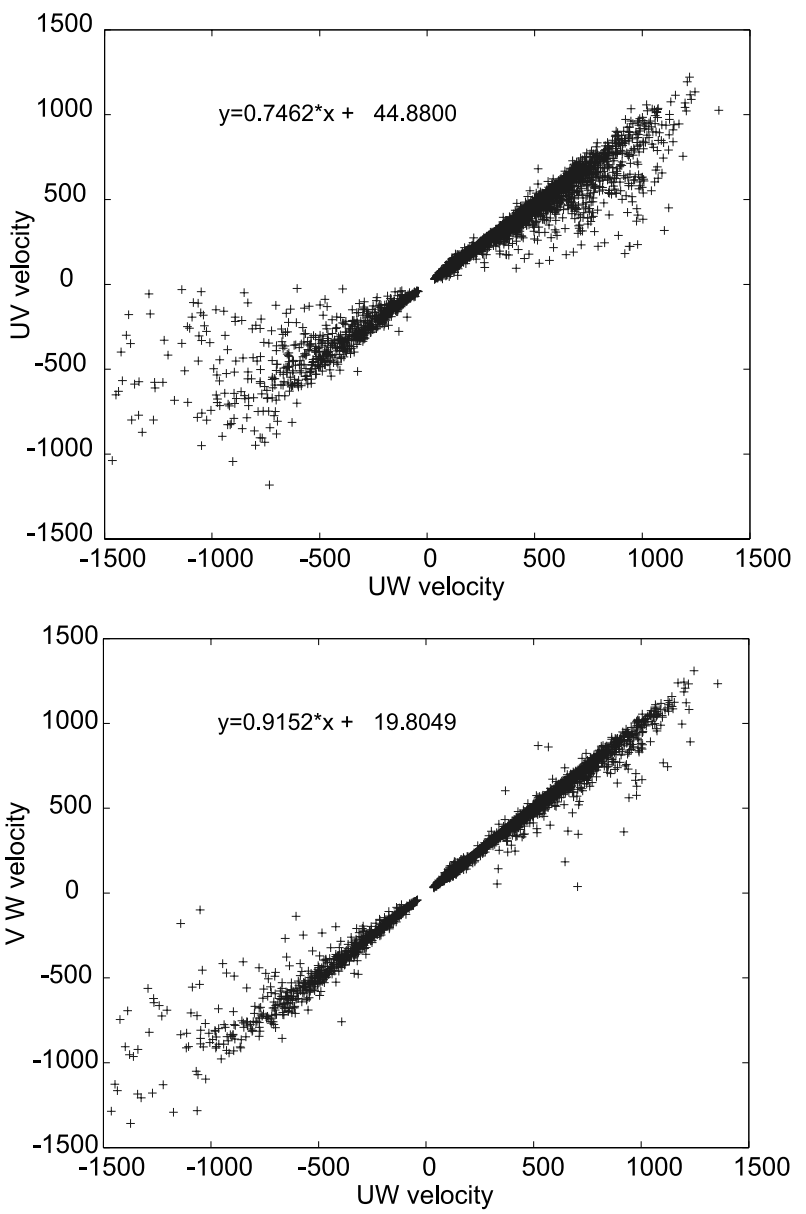

Figure 3. A comparison of scintillation fade pattern true velocities $(\mathrm{m} / \mathrm{s})$ measured using the $\mathrm{U}-\mathrm{V}$ and $\mathrm{V}-\mathrm{W}$ receivers pairs compared to the $\mathrm{U}-\mathrm{W}$ receiver pair. The linear equations in each panel show the result of a least squares fit to the data, which should be a straight line of slope 1 and intercept 0 .

large vertical motions of irregularities within developing spread $F$ bubbles mapping into the horizontal direction [Kil et al., 2000, 2002; D. Hysell, personal communication, 2002]. Each panel of Figure 3 contains about 10,214 points, of which the vast majority are less than $500 \mathrm{~m} / \mathrm{s}$. Since the three receiver pairs should be measuring the same velocities, both examples should show a straight line with a slope of 1 and an intercept of 0 . The least squares fit coefficients of a straight line are shown in each panel. The results are adequate to proceed but disappointing. The U-V receiver pair form the shortest baseline and yield the coefficients in most disagreement with those expected. Some of the divergent values can be attributed to the short baseline. Fortunately, these results can be made more rigorous. As a result of the fade properties demonstrated later in this paper, we will show in the conclusions how to select data that yield an excellent fit.

\section{Velocity Measurements From the Drifting Scintillation Fade Patterns}

[16] Ionospheric drift velocities at the magnetic equator and the anomalies are controlled by tidal winds and dynamo electric fields. During the day the $F$ region is driven westward through coupling to the $E$ region and neutral winds. During the night the $E$ region conductivity decreases, releasing the $F$ region to drift eastward. This typical pattern has been established at the equator with incoherent scatter measurements [Woodman, 1972; Fejer et al., 1981], using satellite measurements of electric field [Aggson et al., 1987; Coley and Heelis, 1989], observing the drift of total electron content depletions [Abdu et al., 1985b], following the movement of ionospheric structure in airglow images [Mendillo and Baumgardner, 1982; Sobral and Abdu, 1991; Tinsley et al., 1997; Mendillo et al., 1997], and by observing the drift of scintillation fade patterns from geostationary satellite signals [Abdu et al., 1985a, 1987; Basu et al., 1991, 1996; Valladares et al., 1996]. More recently, Kil et al. [2000] have used GPS satellite signals to determine ionospheric drifts. The typical pattern of equatorial $F$ region ionospheric drifts has been established beyond doubt. However, there exist departures from this typical pattern during periods of magnetic storms that are not understood but nonetheless produce, for example, brief periods of westward drift at night. Our goal here is not to infer ionospheric drifts but rather to establish the characteristic features of scintillation fade pattern drifts observed by ground-based or potentially airborne GPS receivers. Ionospheric drifts and fade drifts differ by the GPS signal puncture-point velocity.

[17] Briggs et al. [1950] have pointed out that the naïve approach to measuring the drift speed of scintillation fade patterns is to develop the cross-correlation function between two receivers separated by a distance $\zeta_{0}$. The time lag, $\tau_{0}$, is determined from the shift of the cross-correlation function's maximum value. Then the drift speed is just given by $\zeta_{0} / \tau_{0}$. This is called the "apparent velocity." On the other hand, the scintillation fade pattern is also evolving with time, which makes the definition of speed more complex. Another legitimate definition of speed is that given by the motion of an observer in which the scintillation fade pattern evolves most slowly in time or, in the words of Briggs et al. [1950], "the velocity of an observer who has so adjusted his motion over the ground that he experiences the slowest possible speed of fading." This velocity is called the "true velocity" and, in general, it is not the same as the apparent velocity. 


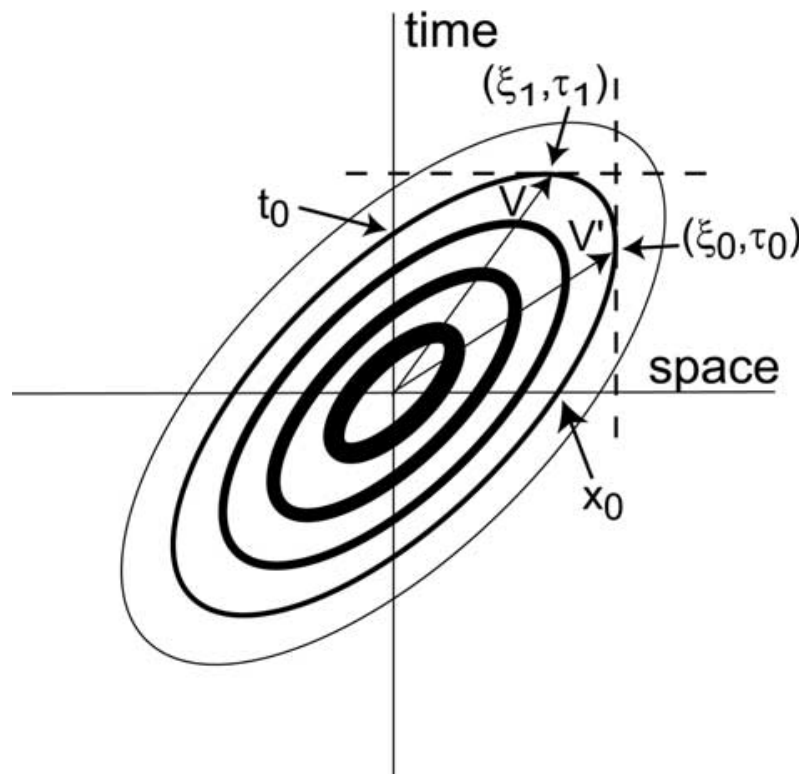

Figure 4. Schematic of a correlation surface in one spatial dimension and time [after Briggs et al., 1950]. Shown are loci of constant correlation value which form ellipses centered on the origin. The correlation values of each ellipse decrease monotonically from the origin. A cross-correlation function is given by the values of the correlation surface along a vertical cut. For example, the cross-correlation function at location $\xi_{0}$ is given by the values of the correlation surface along the vertical dashed line and the maximum in the cross-correlation function is found at time $\tau_{0}$. The autocorrelation function is given by a vertical cut from the origin and has a maximum of one at zero time lag.

[18] Figure 4 illustrates the difference between the true and apparent velocities. Shown are correlation surfaces in one spatial dimension and time. In this representation the correlation surfaces are assumed to be ellipses. At the origin the value of the correlation surface is one and it monotonically decreases away from the origin in both space and time. Correlation function values are given by making a vertical cut in this space. For example, the autocorrelation function is given by the correlation surface values corresponding to a vertical cut through the origin. The cross-correlation function at the spatial location $\zeta_{0}$ is given by the correlation surface values found on the vertical cut intersecting the spatial axis at $\zeta_{0}$. The maximum value of this cross-correlation function occurs at time $\tau_{0}$, so in this representation the apparent velocity is just given by motion along the arrow $V^{\prime}$.

[19] The true velocity is given by the motion of the observer in which the scintillation fade pattern evolves most slowly. In Figure 4 this is represented by the motion of an observer in which the correlation surface decreases most slowly, which is found by determining the spatial separation to yield a maximum in the correlation surface for a given time, that is, the maximum of the correlation surface found along a horizontal cut in Figure 4. To summarize, an observer located at $\zeta_{0}$ will measure a cross-correlation function given by the values of the correlation surface on a vertical cut. The maximum of the cross-correlation function will occur at time lag $\tau_{0}$ and the apparent velocity will be given by $\zeta_{0} / \tau_{0}$. At the point $\left(\zeta_{0}, \tau_{0}\right)$ the correlation surface has a value $\rho$. All of the points on the correlation surface with a value $\rho$ form an ellipse. Following this ellipse around to the point of maximum time delay, the time $\tau_{1}$ is determined with spatial separation $\zeta_{1}$. The true velocity is then given by motion along the arrow $V$ where, for a given value of $\rho$, the maximum time delay or lag occurs. For purposes of the calculation below we also need to define the time delay $\tau_{0}$ in order to agree with Figure 4.

[20] Using straightforward geometrical arguments and assuming that the surfaces of constant cross-correlation amplitude can be represented by ellipses, Briggs et al. have shown that the true velocity is related to the apparent velocity by

$$
V=\frac{V^{\prime}}{1+t_{0}^{2} / \tau_{0}^{2}},
$$

where $V^{\prime}$ is the apparent velocity and $t_{0}$ is the time at which the autocorrelation function takes on the peak value of the cross-correlation function. Briggs et al. also define a characteristic velocity, which is a measure of the scintillation fade pattern change rate in the true velocity reference frame and is given by

$$
V_{C}=\frac{V^{\prime}}{1+\tau_{0}^{2} / t_{0}^{2}}=\frac{x_{0}}{\tau_{0}}
$$

where the denominators of the two equations above are slightly different. The space shift, $x_{0}$, for equal values of correlation is defined as shown in Figure 4. One may understand the characteristic velocity by noting that $V / V_{C}=\xi_{1} / x_{0}$. The space shift $x_{0}$ will scale with Fresnel length. Hence, within a constant, $V / V_{C}$ is the ratio of distance traveled at the true velocity compared to the Fresnel length for equal values of correlation. Alternatively, the ratio $t_{0}^{2} / \tau_{0}^{2}$ determines the extent to which the apparent and true velocities are equivalent. If $t_{0}^{2} / \tau_{0}^{2} \ll 1$, the apparent and true velocities are nearly equal and the ellipses in Figure 4 are greatly elongated along the diagonals represented by the two velocity arrows. On the other hand, if $t_{0}^{2} / \tau_{0}^{2} \cong 1$, then the ellipses are elongated along the time axis and the scintillations measured by a stationary receiver are mostly produced by random fluctuations in the scintillation fade pattern. Finally, if $t_{0}^{2} / \tau_{0}^{2} \cong 1$, then the ellipses are similar to those 

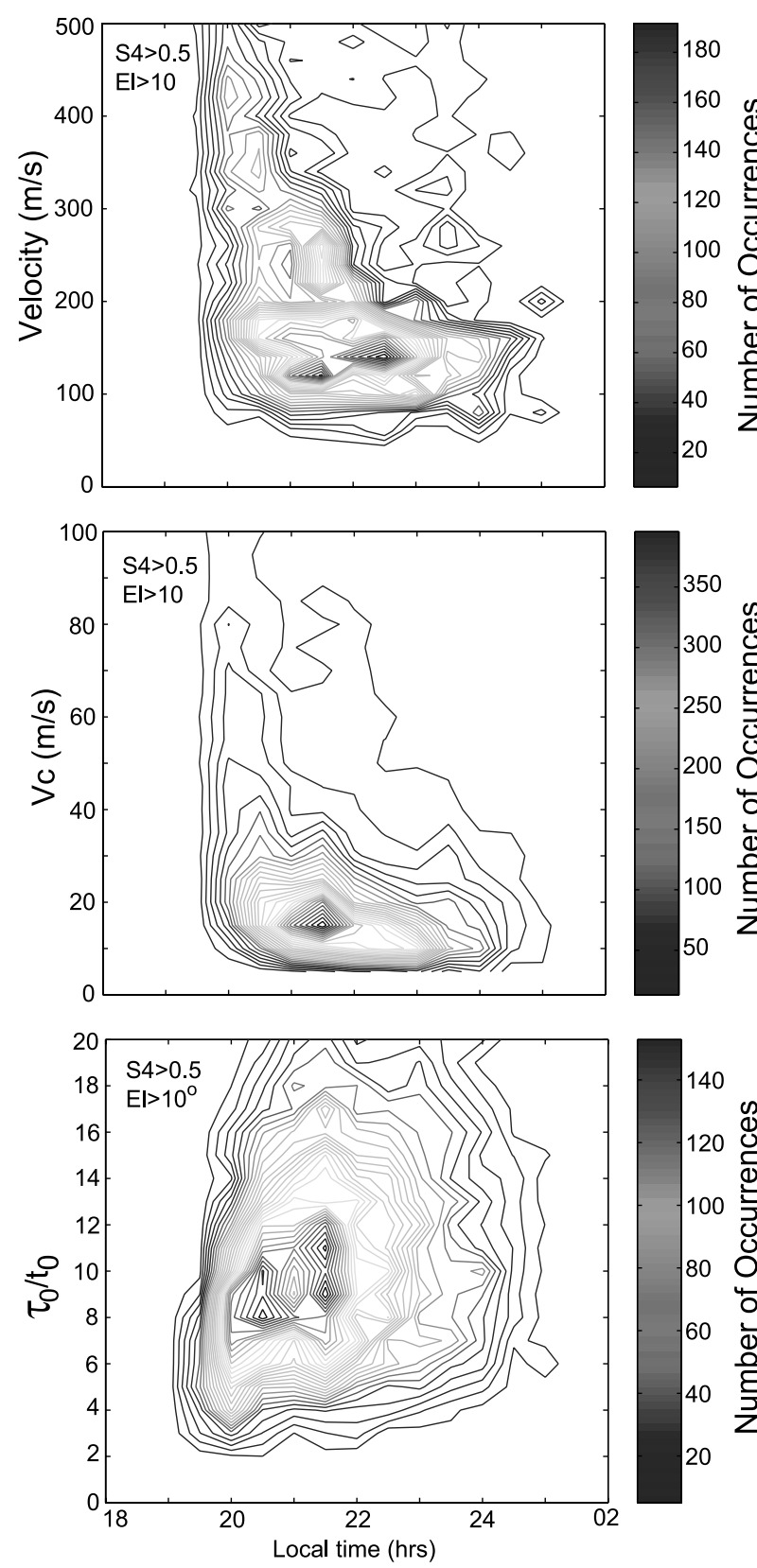

Figure 5. Contour plots of occurrence rates for the true eastward velocity (top), the characteristic velocity (middle), and the ratio $\tau_{0} / t_{0}$ (bottom) versus local time. Shown are the number of occurrences of true velocity in bins of $0.5 \mathrm{~h}$ by $25 \mathrm{~m} / \mathrm{s}$ (top), the number of occurrences of characteristic velocity in bins of $0.5 \mathrm{~h}$ by $5 \mathrm{~m} / \mathrm{s}$ (middle), and the number of occurrences of the ratio $\tau_{0} / t_{0}$ (bottom) in a bin of $0.5 \mathrm{~h}$ by 1 of the ratio. See color version of this figure at back of this issue. shown in Figure 4, and including the corrections (arising from random changes in the scintillation fade pattern) to calculate the true velocity from the apparent velocity is required.

[21] Figure 5 shows scintillation fade pattern eastward velocity occurrence rates estimated from the U-W receiver pair, separated by $715 \mathrm{~m}$, as a function of local time and for positive (eastward) velocities only. The top panel displays the true velocity values, the middle panel displays the characteristic velocity values, and the lower panel displays the values of $\tau_{0} / t_{0}$ observed. Beginning with the top panel, 9307 values of true velocity estimated from a 40 -sec cross-correlation function have been represented in a contour plot of the number of occurrences in bins with dimensions of $0.5 \mathrm{~h}$ by $25 \mathrm{~m} / \mathrm{s}$. The vast majority of the true velocity measurements fall between 100 and $200 \mathrm{~m} / \mathrm{s}$. However, in the early evening there is a tail toward larger velocities in the occurrence distribution and the upper border of the occurrence distribution decreases in velocity as the night progresses. The lower border of the occurrence distribution is nearly constant at about $100 \mathrm{~m} / \mathrm{s}$. This may be the influence of the GPS signal $F$ region puncture-point velocity, which typically adds several tens of $\mathrm{m} / \mathrm{s}$ to the ionospheric drift velocity [Kintner et al., 2001].

[22] This occurrence distribution in the middle panel is calculated from the same correlation functions used in the top panel and is represented as a contour plot showing the number of occurrences in bins of dimensions $0.5 \mathrm{~h}$ by $5 \mathrm{~m} / \mathrm{s}$. The principal feature of this plot is that the characteristic velocities are much smaller than the true velocity, implying that most of the scintillating amplitude fluctuations observed by a stationary receiver are the result of a translating but otherwise static scintillation fade pattern. The corrections to the apparent velocity required to yield the true velocity are small. Furthermore, the random changes in a scintillation fade pattern produced by the evolving ionospheric diffraction screen are small. This fact is emphasized in the lower panel where the occurrence distribution of $\tau_{0} / t_{0}$ is shown. The bulk of the distribution (89\%) is found at values of $\tau_{0} / t_{0}>5$ where corrections to the apparent velocity from random scintillation fade pattern changes, yielding the true velocity, are about $4 \%$ or less. Hence one would expect that a receiver moving at the true velocity (equation (2)) would experience much longer fades than those experienced by a stationary receiver.

[23] The occurrence densities may be converted to probability densities by dividing the values in Figure 5 by the total number of measurements possible in a period of 0.5 hours over the observing period of 45 days. For a single satellite this is 2025 possible correlations. The initial data analysis did not record the number of satellites visible during each $40 \mathrm{~s}$ period in which a correlation could be made, but typically 8-10 satellites were 


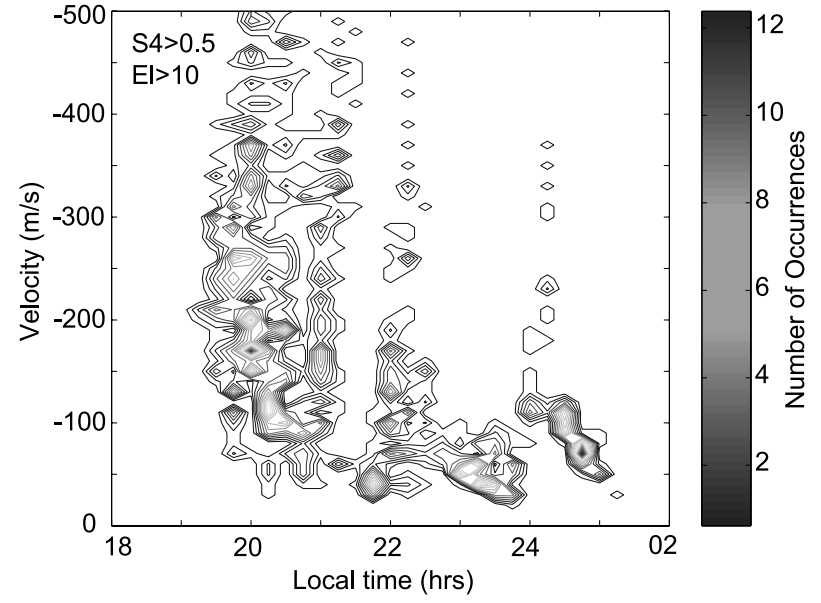

Figure 6. Contour plots of the number of occurrences of negative (westward) true velocity in bins of $0.25 \mathrm{~h}$ by $25 \mathrm{~m} / \mathrm{s}$ versus local time. See color version of this figure at back of this issue.

visible. Assuming that 9 satellites were visible on average and noting that the largest occurrence densities were about 200 per bin (top panel), the highest probability represented in Figure 5 is 0.011 per bin per satellite. Alternately, for all satellites in view, the largest probability represented in Figure 5 (top panel) is about 0.1 per bin.

[24] The occurrence distribution of negative (westward) true velocities as a function of local time is shown in Figure 6. The distribution is represented as a contour plot of the number of occurrences in bins with dimensions of $0.25 \mathrm{~h}$ by $25 \mathrm{~m} / \mathrm{s}$. The distribution of negative velocities has several differences when compared to the distribution of positive velocities. First, there are far fewer occurrences of negative velocities: 907 compared to 9307 positive velocities measured. Next, the negative velocity distribution is more episodic, with individual events dominating the distribution and producing the apparent vertical "stripes." Like the positive velocity distribution, the first few hours after sunset are variable with the distribution displaying an extended tail toward larger negative velocities. However, the distribution over the course of the night shows a large spread from $25 \mathrm{~m} / \mathrm{s}$ to $200 \mathrm{~m} / \mathrm{s}$. We conclude from this distribution that negative (westward) velocities are more likely to be measured during periods when spread $F$ is developing or during brief periods of magnetic activity [see, e.g., Kil et al., 2000, Figure 5; Sobral et al., 2001].

[25] The eastward velocity of scintillation fade patterns shown in the preceding figures yields a one-dimensional picture of velocities for comparison with aircraft velocities (typically 50 to $200 \mathrm{~m} / \mathrm{s}$ ) and for evaluating the possibility of velocity matching or resonance. While the scintillation fade pattern velocity may be primarily onedimensional, aircraft velocities are two-dimensional, in general, having as much of a north-south component as an east-west component. So, to consider how a moving receiver may resonate with a scintillation fade pattern, we also need to evaluate the shape and orientation of the scintillation fade patterns, as is described next.

\section{Shape and Orientation}

[26] GPS scintillation fade patterns are produced when ionospheric irregularities produce a diffraction screen with structure at the Fresnel length. Since the irregularities are believed to be organized by the magnetic field, the scintillation fade patterns should reflect this organization. Optical measurements clearly show the structure of long scale-length $(>10 \mathrm{~km})$ irregularities following the magnetic field [Mendillo and Baumgardner, 1982]. This belief suggests that the scintillation fade patterns should be greatly elongated and aligned in the magnetic north-south direction. These are the two properties that we examine next, beginning first with the subject of orientation and ending with the subject of elongation.

[27] Figure 7 demonstrates how the scintillation fade patterns are organized, using a simple model of a flat earth and the idea that the fades are similar to the shadow of a picket fence cast in the direction of the GPS signal. This view shows the projection of a magnetic field line along the path of a GPS satellite signal onto the hori-

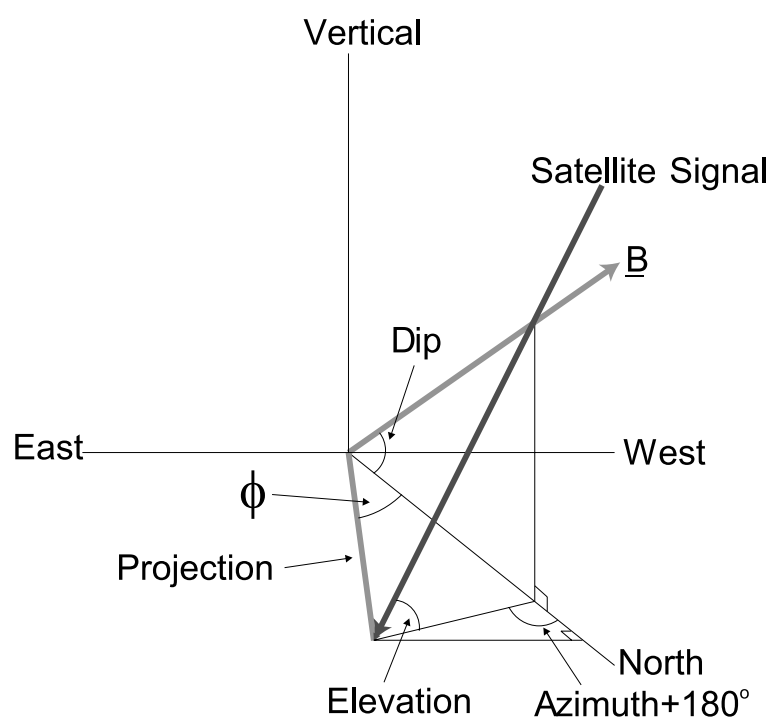

Figure 7. Diagram showing how the magnetic field line can be projected along the signal path into the horizontal plane. The angle $\phi$ is the projection angle between the projected magnetic field and magnetic north. 


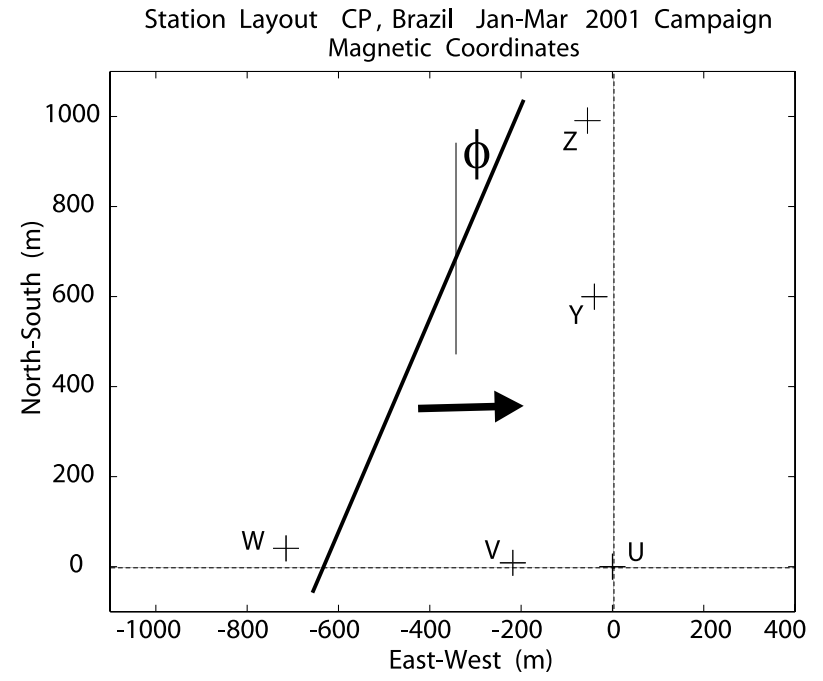

Figure 8. Schematic diagram showing a scintillation fade pattern surface with projection angle $\phi$ moving eastward across the receiver array.

zontal plane, again assuming a flat earth. The projection angle $\phi$ is the angle between the projection of the magnetic field onto the horizontal plane and magnetic north. The projection angle $\phi$ is a function of the magnetic dip angle, $D$, and elevation $(E)$ and azimuth $(A)$ of the satellite given by

$$
\phi=\arctan \left[\frac{-\tan (D) \operatorname{cotan}(E) \sin (A)}{1-\tan (D) \operatorname{cotan}(E) \cos (A)}\right] .
$$

[28] The projection angle $\phi$ can also be measured using the $\mathrm{U}, \mathrm{W}$, and $\mathrm{Z}$ receivers. Figure 8 shows the eastward drift of a scintillation fade pattern surface in schematic form. The projection angle $\phi$ can be calculated by knowing the separation of the $\mathrm{U}, \mathrm{W}$, and $\mathrm{Z}$ receivers and the lag times in the U-W optimal cross-correlation and the U-Z optimal cross-correlation. If the east-west distance between $\mathrm{U}$ and $\mathrm{W}$ is $l_{W U}$ and the north-south distance between $\mathrm{U}$ and $\mathrm{Z}$ is $l_{Z U}$, the relationship between the projection angle $\phi$ and the lag times becomes

$$
\frac{\tau_{Z U}}{\tau_{W U}}=\tan (\phi) \frac{l_{Z U}}{l_{W U}} .
$$

Surprisingly, this relation holds even if the drift velocity has a north-south component (see Appendix A). Small corrections must be accounted for, since the WUZ triplet does not form an exact $90^{\circ}$ angle and we have accounted for these in the calculations that follow, although they make no perceivable difference in the results.

[29] To test this simple model for explaining why scintillation fade patterns are not aligned in the north- south direction, we have compared the calculated projection angle (equation (4)) with the measured projection angle in Figure 9. If the model is valid, the locus of points in Figure 9 should be arranged on a line of slope 1 and intercept 0 , shown as a red line. The top panel shows the results of comparing all points passing the test of $\mathrm{S} 4>0.5$ and elevation angles greater than $10^{\circ}$. The graph strongly suggests that the model can explain some of the data but there are large departures from the model that are not easily explained. We examined both the possibility of vertical flows and the effects of a spherical earth, which could not explain the pattern observed. One factor does account for a substantial fraction of the anomalous results. For signal paths closely aligned with the magnetic field direction, the simple projection model fails.

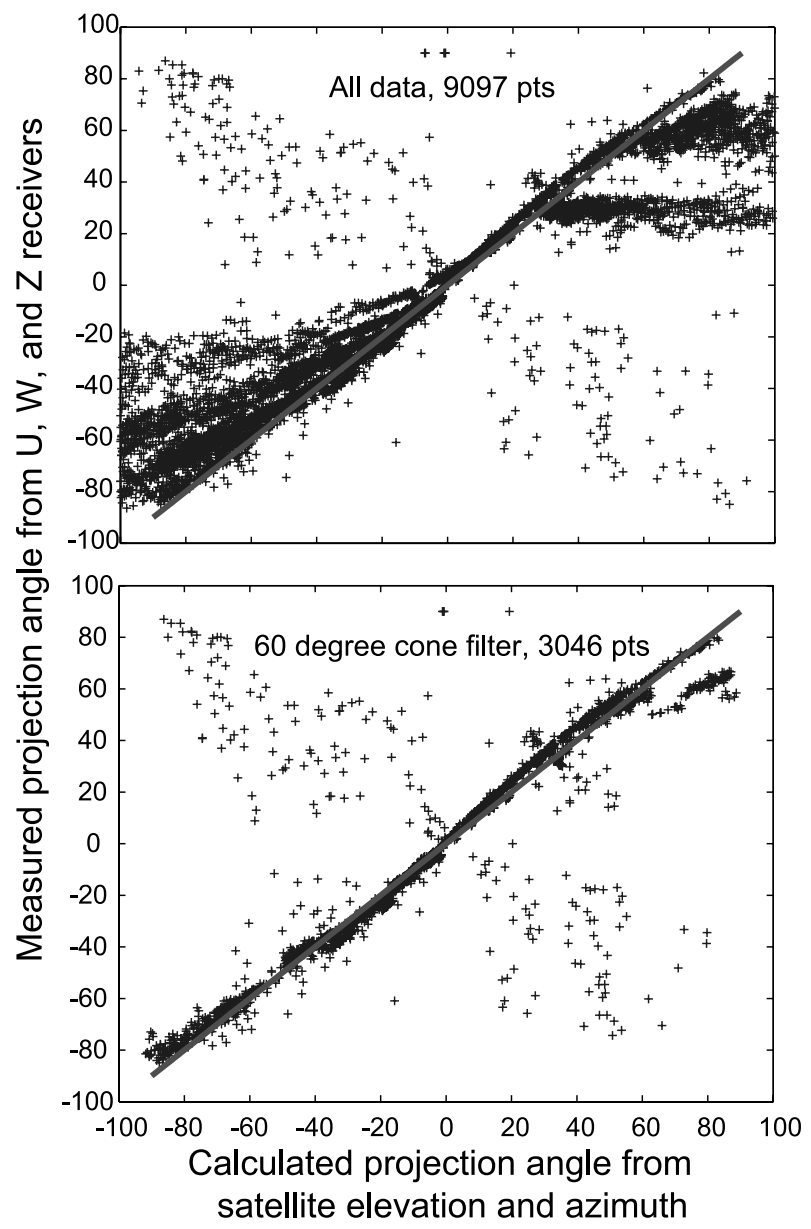

Figure 9. A comparison of calculated and measured projection angles. (top) All available data are shown. (bottom) Only data for signal paths separated by at least $60^{\circ}$ from the magnetic field direction are shown. The red line is the expected result for the projection model. See color version of this figure at back of this issue. 
This leads to the lower panel where the distribution of measured projection angles agrees very well with calculated projection angles. In the lower panel we have eliminated all points where the signal path was aligned within $60^{\circ}$ of the magnetic field. This is a large portion of the sky, although much of the eliminated solid angle is below the horizon. It reduces the number of data points to one-third of the value in the upper panel, which can be partially understood by noting that northward field lines map to lower altitudes at the equator and are more likely to participate in equatorial spread $F$ than the southward field lines. The set of points roughly perpendicular to the red lines in Figure 9 represent less than $0.5 \%$ of the data and are not considered significant.

[30] The somewhat arbitrary choice of $60^{\circ}$ to create a cone around the magnetic field of eliminated data produces a set of projection angle values that agree astoundingly well with more than $90 \%$ of the points close to the predicted line. Although not shown, if the cone half-angle is reduced to $50^{\circ}$, a substantial number of points do not agree well. The value of $60^{\circ}$ cannot be significantly smaller. In another study, not shown here, the satellite signal directions were examined for cases where the measured projection angle either agreed or disagreed with the calculated projection angle. The test for agreement was that the measured and calculated projection angles agreed within a factor of $20 \%$. With this test the data set was divided almost evenly between agreement and disagreement. When the elevation and azimuths of the disagreeing signal paths were plotted, they were not distributed smoothly over the $60^{\circ}$ cone around the magnetic field. Instead they were contained within the $60^{\circ}$ cone and weighted toward low elevations $\left(<40^{\circ}\right)$. At this juncture we have no compelling explanation for either the existence or the distribution of the disagreeing signal paths. We simply conclude that the flat-earth projection model explains the observed results over the majority of the sky that is farther from the magnetic field direction.

[31] Given the observation that scintillation fade patterns are usually not aligned north-south, the exception being when the signal azimuth is the same as the magnetic azimuth or $180^{\circ}$ from the magnetic azimuth, we can now examine the question of elongation; that is, how long are the fade surfaces? Since the scintillation fade patterns are evolving in their own reference frame, examining $U-Z$ cross-correlation functions is only a valid test of elongation when the scintillation fade pattern is aligned in the north-south direction parallel to the U-Z line, that is, when the projection angle is 0 . We have examined this hypothesis by plotting the mean value of the optimal cross-correlation magnitude in $5^{\circ}$ bins as a function of the projection angle and have used all data, including those collected from signals inside the $60^{\circ}$ half-angle cone around the magnetic field direction. The result is shown in Figure 10, along with the standard

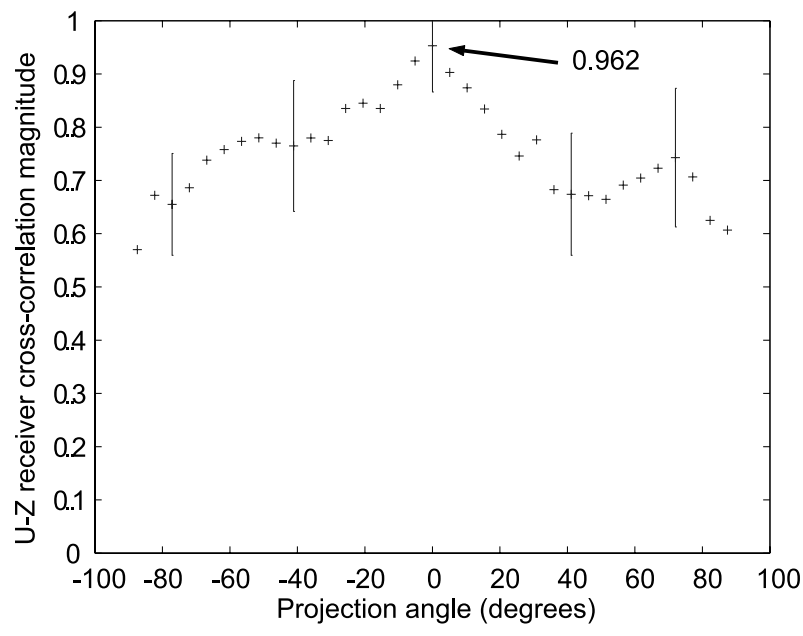

Figure 10. The means of optimal cross-correlation values between the $U$ and $Z$ receivers as a function of the projection angle. The vertical lines represent standard deviations at several different projection angles.

deviations of the mean values at several points. As expected, the mean optimal cross-correlation values are largest at the $0^{\circ}$ projection angle with a curious elevation in the means between $+70^{\circ}$ and $+80^{\circ}$ projection angles. The mean value of the optimal cross-correlation values corresponding to projection angles of 0 (actually within $2.5^{\circ}$ of 0 ) is 0.962 and the standard deviation of this value is only 0.045 . Hence over the $991 \mathrm{~m}$, north-south distance between the $\mathrm{U}$ and $\mathrm{Z}$ receivers, we do not believe that we have detected any change in the scintillation fade pattern. This implies that the scintillation fade patterns are greatly elongated, more than $10 \mathrm{~km}$ and perhaps more than $100 \mathrm{~km}$. This is certainly correct for scintillation fade patterns with small projection angles. The question of whether it is also correct for scintillation fade patterns with larger projection angles is interesting and can be addressed using other receiver pairs but again, this is the subject of a future investigation.

\section{Fade Width}

[32] The width of the scintillation fades to first order is scaled by the Fresnel radius, $\sqrt{2} r_{F}=\sqrt{2 \lambda d}$, where $\lambda$ is the GPS wavelength of $19 \mathrm{~cm}$ and $d$ is the distance to the scattering volume, which can be derived simply from a consideration of two-slit diffraction. A more rigorous consideration [Salpeter, 1967] demonstrates that, for weak scattering, the wave number spectrum of the scintillations takes on the form

$$
\Phi_{L}(k)=4 \Phi_{\phi}(k) \sin ^{2}\left(\frac{k^{2} r_{F}^{2}}{4 \pi}\right),
$$


where $\Phi_{\phi}(k)$ is the power spectral density of the scattering irregularities. Since $\Phi_{\phi}(k)$ generally decreases with increasing $k$, usually only the first peak in $\Phi_{L}(k)$ is significant, although it may be weighted toward somewhat smaller values of $k$. In the absence of this skew, the dominant width, $L$, in the wave number spectrum occurs when

$$
\frac{k^{2} r_{F}^{2}}{4 \pi}=\frac{\pi}{2}
$$

or at the value $L=\sqrt{2} r_{F}=\sqrt{2 \lambda d}$. If the one-dimensional scintillation fade pattern were a sinusoidal function, $L$ would be the distance between either peaks or nulls. Once again, since the wave number spectrum will be weighted somewhat toward smaller values of $k$, we expect that $L \leq \sqrt{2 \lambda d}$.

[33] The concept of a Fresnel radius suggests that it should be possible to discover a single organizing scale length in our data after scaling for the varying distances to the ionospheric puncture points and the fade orientation. We call this the normalized Fresnel length. We measure the width of the scintillation fade pattern by first measuring the width in time of the autocorrelation function at half maximum and multiplying the result by the true velocity deduced from the U-W receivers. This yields a length that is proportional to the scintillation fade pattern width. If the autocorrelation function was a co-sinusoid, the half-maximum width would represent $1 / 3$ of the distance between fade peaks (or nulls), which is equivalent to $L$, so we multiply again by a factor of three [Kintner et al., 2001]. This yields a measure of the scintillation fade pattern width. To estimate the normalized Fresnel length, this width must be adjusted for the slant range to the scattering volume and for the fade orientation.

[34] The results of this analysis can be found in Figure 11. The adjustments are shown one step at a time so the reader can weigh the effect of each correction. Figure 11a shows the results, as a function of local time, of using all of the U-W data to calculate the pattern width and shows a broad distribution. The calculated widths are distributed primarily between $400 \mathrm{~m}$ and $800 \mathrm{~m}$ but there is a long tail in the distribution extending to more than $1500 \mathrm{~m}$. Figure $11 \mathrm{~b}$ shows the results of adjusting for the slant range distance using an assumed altitude of $350 \mathrm{~km}$ and a slant factor of $\sin \left(E^{\prime}\right)$ where $E^{\prime}$ is the satellite elevation at the scattering location. This can be easily estimated for a spherical earth and is found in textbooks [Hofmann-Wellenhof et al., 2001]. The correction for slant range condenses the distribution somewhat, confining the bulk of the measurements to between $400 \mathrm{~m}$ and $650 \mathrm{~m}$. In Figure $11 \mathrm{c}$ the results of adjusting for orientation or projection angle $(\cos (\phi))$ are shown. In this case the body of the distribution has been narrowed
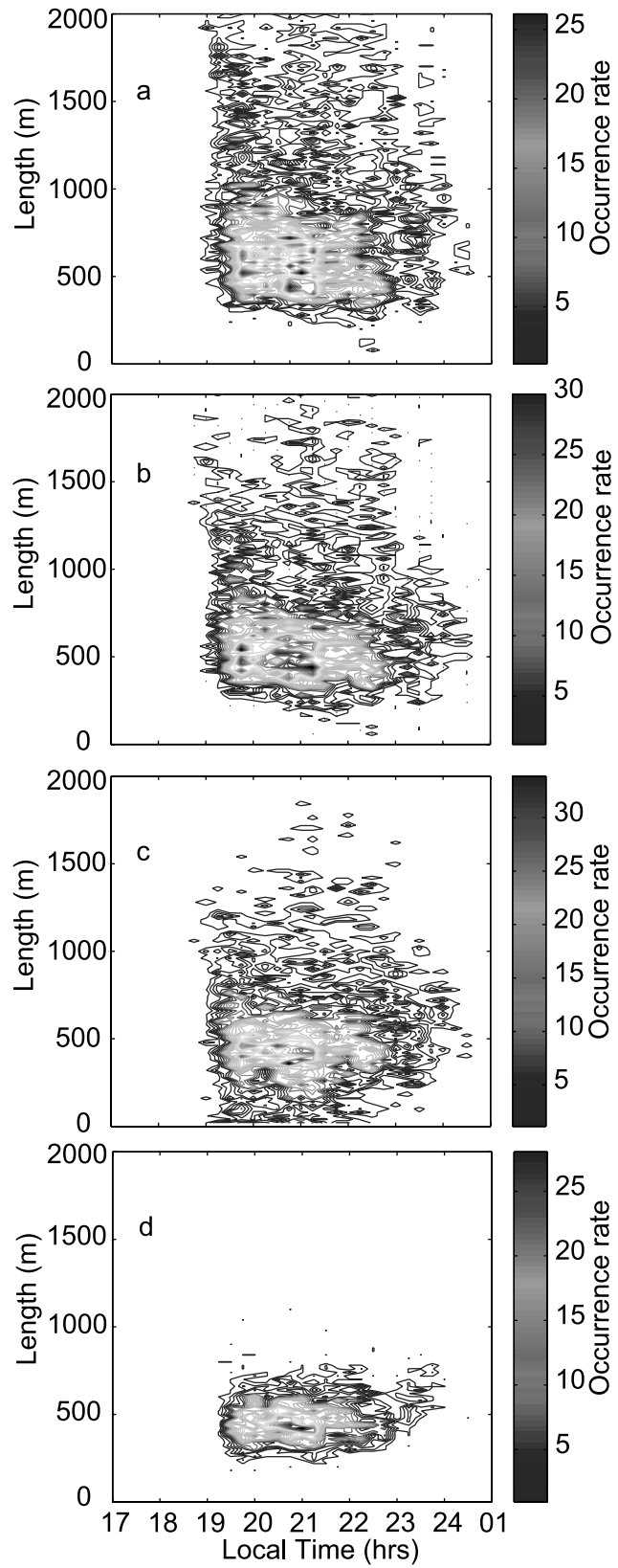

Figure 11. Contour plots of occurrence rates for different methods of estimating the measured Fresnel length. (a) Makes no adjustments. (b) Adjusts for slant range only. (c) Adjusts for slant range and projection angle. (d) Adjusts for slant range and projection angle and only uses data whose signal paths are separated by at least $60^{\circ}$ from the magnetic field direction. See color version of this figure at back of this issue. 


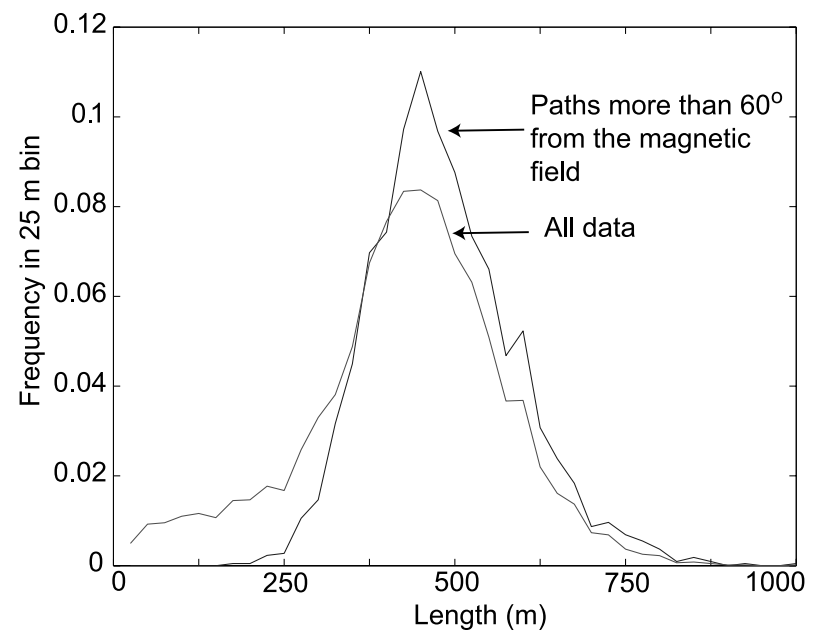

Figure 12. Probability distributions for the measured fade width within $25 \mathrm{~m}$ bins. Shown are the distributions reduced from Figures 11c and 11d. The red curve labeled "all data" corresponds to Figure 11c where the fade widths have been adjusted for slant range and orientation. The blue curve labeled "paths more than $60^{\circ}$ from the magnetic field line" corresponds to Figure 11d and includes all data adjusted for slant range and orientation with signal paths aligned at least $60^{\circ}$ from the magnetic field direction. See color version of this figure at back of this issue.

substantially to between $400 \mathrm{~m}$ and $500 \mathrm{~m}$. The tail in the distribution at long lengths has reduced substantially and a new tail at shorter lengths has been introduced. The tail at shorter lengths arises from previously over-estimating the fade length at large projection angles. Figure 11d shows the results after eliminating examples where the satellite signal paths were aligned within $60^{\circ}$ of the magnetic field. Now there is a narrow, well-defined peak without elongated tails, as expected if the fade width truly is organized by the normalized Fresnel length and originates from a restricted range of altitudes. Some of the remaining distribution width likely results from varying altitudes for the scattering volume and some is likely produced by different power spectrums of the scattering irregularities. The factor of 3 relating the autocorrelation half width to the full width of the scintillation fade pattern will also vary, depending on the shape of the horizontal wave number spectrum, and may be a lower bound.

[35] To determine the most likely fade width, we have examined the distribution of fade widths for the conditions corresponding to Figures $12 \mathrm{c}$ and $12 \mathrm{~d}$; that is, the fade widths have been corrected for the elevation and projection angle for the former case while additionally eliminating signal paths inside a $60^{\circ}$ half-angle cone around the magnetic field direction in the latter case. The result is shown in Figure 12 where the distribution normalization factor is set to make the area under the distribution equal to 1 . The two distributions are remarkably similar, with the most likely estimate of the normalized Fresnel length being about $450 \mathrm{~m}$. The principal effect of removing examples where the signal path is within $60^{\circ}$ of the magnetic field is to eliminate a tail in the distribution at short lengths (less than $300 \mathrm{~m}$ ).

\section{Duration of Scintillation Fade Patterns}

[36] The time duration of ionospheric scintillation fade patterns is dependent on the reference frame of the receiver. For a fixed receiver the time duration is the time shift required to produce a given correlation value. If the reference frame is moving so that there is also a space shift, then the time lag at optimal cross-correlation yields a measure of the fade duration. The fade duration then becomes a function of the cross-correlation value at optimal lag. Hence the investigation of scintillation fade pattern duration implies investigating the time evolution of optimal cross-correlation values. In Figure 4 the time shift for a given correlation value $\rho$ and a fixed receiver is $t_{0}$. For a moving receiver, the reference frame producing the largest time shift for the same correlation value is moving with the true velocity given by $\zeta_{1} / \tau_{1}$. Thus, to investigate the duration of scintillation fade patterns in a resonant or velocity-matching time frame implies investigating the evolution of the optimal cross-correlation values of a receiver moving at the true velocity.

[37] In Figure 5 the relation between the true velocity and the apparent velocity was investigated with the conclusion that they are nearly equal for the data set considered here. This implies that the values $\xi_{1}$ and $\tau_{1}$ are, to a good approximation, the same as $\xi_{0}$ and $\tau_{0}$, which are measured by the spaced receivers. The large values of $\tau_{1} / t_{0}(5-15)$ imply that the ellipses of correlation in Figure 4 are greatly elongated, nearly forming a straight line. Consequently, to a good approximation in equation (2), the evolution of optimal cross-correlation values at the apparent velocity is the same as the evolution at the true velocity. The values of $\xi_{0}$ are the separation distances of $\mathrm{U}, \mathrm{V}$, and $\mathrm{W}$ receivers given in Table 1 and the values of $\tau_{0}$ are found by dividing the values of $\xi_{0}$ by the apparent velocity (or the true velocity). The evolution of the optimal cross-correlation values then can be investigated as a function of optimal time lags to yield the evolution in the true velocity frame.

[38] Figure 13 shows the cross-correlation magnitude occurrence frequencies for the complete data set from the $\mathrm{U}, \mathrm{V}$, and $\mathrm{W}$ receivers with the condition that $\mathrm{S} 4>0.5$ and elevation is greater than $10^{\circ}$. More than 30,000 values of optimal cross-correlation and optimal lags are shown as the logarithmic occurrence distribution, the logarithm of the number of events occurring in a bin of 


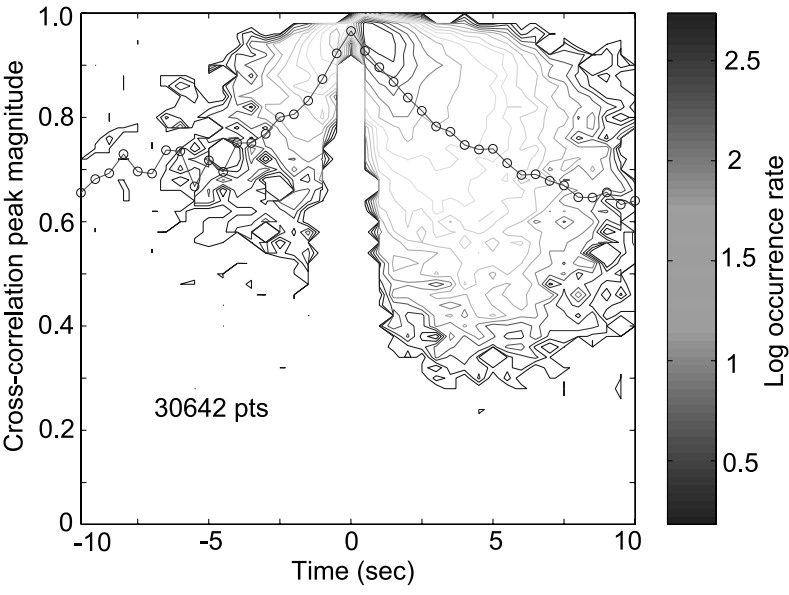

Figure 13. Evolution of the optimal cross-correlation amplitude as a function of time. The logarithm of the occurrence rate in bins of $0.5 \mathrm{~s}$ by 0.02 correlation amplitude are shown on a contour plot. Positive time represents eastward flows and negative time represents westward flows. The mean in each $0.5 \mathrm{~s}$ bin is also plotted. See color version of this figure at back of this issue.

$0.5 \mathrm{~s}$ and 0.02 of correlation magnitude. In addition, the mean value of the cross-correlation values in each $0.5 \mathrm{~s}$ bin is calculated and displayed. Figure 13 illustrates how the correlation values evolve in time in the scintillation fade pattern reference frame or, alternately, how a moving receiver in resonance with the scintillation fade pattern would measure the distribution of correlation values. The pattern is asymmetrical around 0 time because there are far more eastward or positive velocity measurements in the data set; negative time just implies westward drift. For positive times the mean value of the optimal cross-correlation distribution decreases more gradually with increasing time. At $5 \mathrm{~s}$ the mean value is 0.7 but by $10 \mathrm{~s}$ the mean value has only declined to 0.6. The occurrence distribution indicates that many of the measured cross-correlation values exceed the mean and that the most probable values are larger than the mean. Measurements of correlation values exceeding 0.9 are made up to times of at least $7.5 \mathrm{~s}$. By $10 \mathrm{~s}$ there are too few measurements to make meaningful conclusions.

[39] In Figure 14 the distribution of measured correlation values at $5 \mathrm{~s}$ is shown as the number of correlation values in a bin of 0.05 width and normalized to the total number of measurements at $5 \mathrm{~s}$. The most likely correlation value is 0.85 , and $34 \%$ of the values occur at 0.85 optimal correlation or larger. In general, the correlation distributions are skewed toward larger values of optimal correlation. The value of optimal correlation representing significant change in the scintillation fade pattern is arbitrary but for the receiver spacing used in this experiment, the mean optimal correlation value has declined to only 0.6 at $10 \mathrm{~s}$, after which there are too few measurements to reach any conclusions. An additional factor contributes to the decline in the correlation value. The data for cross correlation corresponds to the same UT intervals. A more robust method is to delay the time interval for the "downstream" receiver by the fade transit time between the receivers. Since a $10 \mathrm{~s}$ transit time is a significant fraction of the $40 \mathrm{~s}$ time interval used for correlation, one would expect the correlation value to decrease, even if the fade pattern is completely unchanged during the transit. Hence the correlation values in Figures 13 and 14 are a lower bound, and the true correlation values may be larger.

\section{Discussion and Summary}

[40] By measuring correlation lag values between multiple receivers, the detailed characteristics of GPS L1 C/A amplitude scintillations have been examined under the equatorial anomaly. Generally, these measurements have demonstrated that L1 fades are both spatial and temporal phenomena. In this section the results are placed in context, sources of error are evaluated, and potential opportunities for further investigations are discussed. The organization is parallel to that developed in previous sections where the subjects of velocity, orientation, shape, size, and duration are presented.

[41] The velocity of drifting scintillation fade patterns is the combination of ionospheric drift with the movement of the signal puncture point in the ionosphere. Kintner et al. [2001] demonstrated that most of the eastward GPS signal puncture-point velocities fall be-

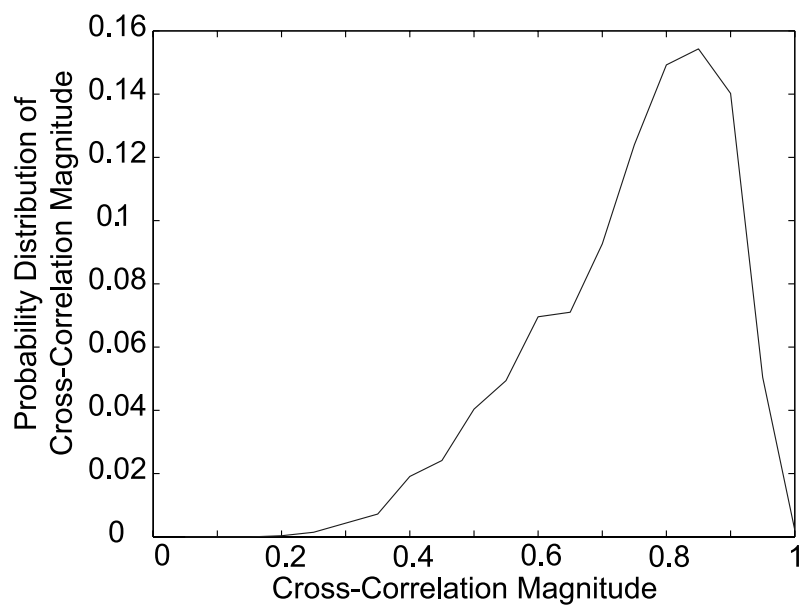

Figure 14. Probability density of optimal cross-correlation amplitude at $5 \mathrm{~s}$ time duration. The normalized occurrence rate is plotted for bins of 0.05 . 
tween $-100 \mathrm{~m} / \mathrm{s}$ and $300 \mathrm{~m} / \mathrm{s}$ with a small number outside this range. Previous measurements of ionospheric drift velocities suggest that a range of typical nighttime values is from $50 \mathrm{~m} / \mathrm{s}$ to $130 \mathrm{~m} / \mathrm{s}$, although smaller and substantially negative values can be recorded during periods of magnetic activity [see, e.g., Kil et al., 2000, and references therein]. This range of ionospheric drift velocities is consistent with the results in Figure 5 where the fade drift true velocity is almost completely contained within the range of $100 \mathrm{~m} / \mathrm{s}$ and $300 \mathrm{~m} / \mathrm{s}$. Some measurements lie outside this range, particularly for larger velocities and early in the evening when spread $F$ develops. Kil et al. [2000] point out that vertical ionospheric flows can be misinterpreted as horizontal flows by the spaced-receiver technique. Given that irregularities within spread $F$ bubbles may have vertical velocities of $1000 \mathrm{~m} / \mathrm{s}$ or larger (D. Hysell, personal communication), this may lead to the apparently large eastward velocities observed during the developmental phase of equatorial spread $F$. Overall the maximum observed true velocities are consistent with the known values of ionospheric drift, GPS signal puncture-point velocities, and the possible confusion of vertical drifts with horizontal drifts.

[42] The minimum range of velocities is somewhat curious in the top panel of Figure 5 where there is an apparent lack of measurements at less than $50 \mathrm{~m} / \mathrm{s}$. The explanation is that drifts probably exist at these slow speeds but that it is difficult to observe them with a longbaseline receiver pair. At $35 \mathrm{~m} / \mathrm{s}$ it would take more than $20 \mathrm{~s}$ for a scintillation fade pattern to drift from the W receiver to the $\mathrm{U}$ receiver. During this time the scintillation fade pattern will evolve for a variety of reasons discussed earlier. Since we have used a minimum threshold for the optimal cross-correlation value of 0.3 , long delay times between a scattering source shadowing two separated receivers may yield poor correlation values and this data would be omitted.

[43] The negative velocity occurrence rates shown in Figure 6 have a behavior similar to the positive velocities but with far fewer measurements. The range of velocities is similar to the discussion in the previous paragraph but now the distribution is more sporadic and appears to be caused by a few or several discrete events. Slower velocities are discovered in the bin covering $25 \mathrm{~m} / \mathrm{s}$ to $50 \mathrm{~m} / \mathrm{s}$, suggesting that, in at least some cases, optimal cross-correlation amplitudes exceed 0.3 for time lags exceeding $15 \mathrm{~s}$. Again, very low velocities (less than $25 \mathrm{~m} / \mathrm{s}$ ) are not apparent, probably because of the very long drift times associated with the slow velocities.

[44] The middle panel of Figure 5 shows the characteristic velocity. Previous measurements of VHF and UHF frequencies suggest that it should be small compared to the true velocity [Vacchione et al., 1987; Spatz et al., 1988] and that the characteristic velocity should be somewhat larger in the early evening compared to later times [Bhattacharyya et al., 2001]. Kil et al. [2000] conclude that, for the GPS L1 signal, the characteristic velocity is so small that it cannot be measured with a baseline of $79 \mathrm{~m}$ because the receiver noise dominates the decorrelation produced by a changing phase screen. To overcome this problem, the experiment described herein employs a $715 \mathrm{~m}$ east-west baseline between the $\mathrm{U}$ and $\mathrm{W}$ receivers. In Figure 5 the characteristic velocity is a few $10 \mathrm{~s}$ of $\mathrm{m} / \mathrm{s}$ during the development of spread $F$ while at later times during the evening the characteristic velocity drops to $10-20 \mathrm{~m} / \mathrm{s}$. The minimum value of $8 \mathrm{~m} / \mathrm{s}$ likely is set by the receiver noise levels.

[45] In the lower panel of Figure 5 the ratio $\tau_{0} / t_{0}$ is examined to understand how significant the effect of the characteristic velocity is on the apparent velocity or, in other words, whether the apparent velocity is nearly equal to the true velocity. The apparent and true velocities are related by $V^{\prime}=V\left(1+t_{0}^{2} / \tau_{0}^{2}\right)$ so that the ratio $\tau_{0} / t_{0}$ is a measure of how much the apparent and true velocities differ. In the bottom panel of Figure 5 the ratio $\tau_{0} / t_{0}$ as a function of local time is shown as a contour plot of occurrence rate. The ratio increases steadily during the course of the evening as expected since the turbulent development phase of spread $F$ is in the early evening. Nearly $90 \%$ of the ratios occur above a value of 5 with a mean value of about 10 . Hence within a factor of $4 \%$ for $90 \%$ of the measurements, and with a mean difference of about $1 \%$, the apparent and true velocities are equal. For this reason we could employ the apparent velocity in determining the duration of scintillation fade patterns.

[46] Next we considered the orientation and shape of the scintillation fade patterns. The simple projection model was introduced in which the scintillation fade patterns are organized by the projection of the magnetic field line along the signal path onto the horizontal plane, assuming a flat earth. This model worked well if signal paths within $60^{\circ}$ of the magnetic field line were eliminated. Even if all signal paths were included, the projection model provided organization to the signals (top panel of Figure 9), although the departures from the simple model (primarily smaller measured projection angles compared to estimated projection angles) are difficult to explain. A separate study (not shown here) demonstrated that the simple projection model fails primarily at lower elevation angles $\left(<40^{\circ}\right)$ within the $60^{\circ}$ cone about the magnetic field direction. The study also showed that examples agreeing or disagreeing with the simple model were about equal in number. At this point, no specific explanation seems to explain satisfactorily why about half of the measured projection angles do not fit the simple model, and resolution must await further investigation.

[47] With the understanding that scintillation fade patterns are not typically aligned north-south but in a 
direction characterized primarily by the projection angle, especially for angles within $45^{\circ}$ of north-south, the shape of GPS L1 scintillation fade patterns can be examined. The question being addressed is, "Are scintillation fade patterns greatly elongated?" Elongation of the scintillation fade patterns is tested by examining the optimal cross-correlation amplitude for receivers separated along the axis of elongation where the axis of elongation is ordered by the projection angle. Figure 10 shows the result of this test where, as expected, the optimal crosscorrelation amplitude has a maximum of 0.962 within $2.5^{\circ}$ of the zero projection angle. The distribution in Figure 10 was created with 9097 correlations. It did not exclude events where the signal paths were within $60^{\circ}$ of the magnetic field, although one should note that when the satellite signal arrives from the north but at a smaller elevation angle than the magnetic field, the projection angle is near $180^{\circ}$ and these data are not shown. The mean optimal correlation value of 0.962 with a standard deviation of 0.045 is within the resolution of the receivers; that is, the receivers have detected no change in the scintillation fade pattern over a distance of $991 \mathrm{~m}$ when the projected fade lies along the path between the $\mathrm{U}$ and $\mathrm{Z}$ receivers, which is very nearly magnetically north-south. This raises two further questions. Just how elongated are the scintillation fade patterns beyond $1 \mathrm{~km}$, and are these scintillation fade patterns elongated for projection angles other than in the north-south direction? This data set is unable to respond to the first question. In principle, the latter question can be addressed by examining other receiver pairs, such as the $\mathrm{W}$ and $\mathrm{Z}$ receivers; this issue must await a later effort.

[48] The width of the scintillation fade pattern is determined from the product of the autocorrelation function width with the pattern drift velocity. The initial calculation yielded a distribution broader than expected from the naïve consideration that the fade widths are organized by a single scale length, the Fresnel length (see Figure 11). For a stationary observer the distribution of fade widths is primarily between $400 \mathrm{~m}$ and $700 \mathrm{~m}$ with an extended tail up to $2000 \mathrm{~m}$. Furthermore, there is some local time dependence in the distribution which is broader at earlier local times. To investigate the existence of a single organizing scale length, the distribution of fade widths was adjusted for slant range, assuming a constant altitude for the scattering volume and a spherical ionosphere (as described in section 6), and for scintillation fade pattern orientation. This result produced a more compact distribution, with most measurements confined between $400 \mathrm{~m}$ and $500 \mathrm{~m}$, but with distribution tails to both larger and smaller values. Finally, by excluding events whose signal paths were aligned within $60^{\circ}$ to the magnetic field a very compact distribution was produced that was independent of local time and that yielded a well-organized scale length.
[49] The most likely scale length was determined to be about $450 \mathrm{~m}$, assuming that the $3 \mathrm{~dB}$ width of the autocorrelation function is related to the fade width by a factor of 3. The expression for weak scattering [Salpeter, 1967] predicts that the fade width distribution should be maximal near $L=\sqrt{2} r_{f}=\sqrt{2 \lambda d}$. Hence for the L1 wavelength of $19 \mathrm{~cm}$ this yields an altitude of $530 \mathrm{~km}$ for the scattering source. This is somewhat larger than the nominal altitude of an $F$ region peak density of $350 \mathrm{~km}$.

[50] A similar analysis was performed by Rino and Owen [1980] at VHF, UHF, and L-band frequencies using wideband satellite signals received at Kwajalein (see their Figure 10). In their case no adjustment for the projection angle was computed and details of the relation of the signal path to the magnetic field direction are not stated. They are able to show that the estimated Fresnel length divided by the scan velocity yields a lower bound to the autocorrelation half-width. This is equivalent to the estimated Fresnel length being a lower bound to the Fresnel length calculated from the autocorrelation halfwidth and drift velocity. Their plots look similar to Figures $11 \mathrm{a}$ and $11 \mathrm{~b}$ with a large spread to longer lengths or longer autocorrelation half-widths. Since the magnetic dip angle at Kwajalein is about $16^{\circ}$ the distribution of autocorrelation widths to larger than predicted values might be explained by considering finite projection angles.

[51] There are two possible error sources in the estimate of $530 \mathrm{~km}$ : the assumption of a $350 \mathrm{~km}$ altitude scattering layer in creating Figures 11 and 12 and the factor relating the $3 \mathrm{~dB}$ autocorrelation width to the fade width. In the former case the dependence of the measured fade width distribution on an assumed altitude is second order because the altitude assumption is only required for correcting a flat earth geometry to a spherical earth geometry. The measured altitude of the scattering will decrease but by less than $10 \%$. The latter source of error, the relation between the autocorrelation $3 \mathrm{~dB}$ width and the fade width, is more problematic. The factor of 3 used here represents a sinusoidal fade pattern which is probably rare. In a different analysis, Kintner et al. [2001] compared discretely measured velocities on a short baseline with velocities inferred from the $3 \mathrm{~dB}$ width of the autocorrelation function. From that comparison the factor relating the $3 \mathrm{~dB}$ width of the function with the fade width was found to be 3.2 to 4.6. If numbers larger than 3 are assumed for the scaling factor, the fade widths in Figures 11 and 12 become larger and the estimated altitude of the scattering source increases in altitude. The contribution of this study is not the absolute value of the Fresnel length estimated from the width but rather that the fade widths can be reduced to a single well-organized scale length. The absolute relation of that scale length to a Fresnel length and altitude requires a 
different experiment in which irregularity altitudes can be independently measured.

[52] The last investigation analyzed the duration of the scintillation fade pattern in the drifting reference frame. Briggs et al. [1950] point out that there are two reference frames moving at the apparent and true velocities. The true velocity frame is the reference frame in which the signal fades have the longest duration. The analysis herein demonstrated that, for GPS L1 signal fades produced by spread $F$ at the equatorial anomaly, the true and apparent velocities are equal to a very good approximation, within $4 \%$ or better for $90 \%$ of the measurements, so that the reference frames are interchangeable. This allows us to examine the fade duration by examining the optimal cross-correlation amplitudes at the fixed receivers, associating each amplitude with a delay given by the time lag of optimal correlation. This was done for the $\mathrm{U}, \mathrm{V}$, and $\mathrm{W}$ receivers, yielding the distributions in Figures 13 and 14. The result is an optimal correlation pattern that decays somewhat more rapidly before $5 \mathrm{~s}$ duration than after $5 \mathrm{~s}$ duration. After $10 \mathrm{~s}$, the available data are inadequate to draw any conclusions. At $5 \mathrm{~s}$ duration, $49 \%$ of the optimal cross-correlation amplitudes were greater than or equal to 0.8 and $19 \%$ were greater than or equal to 0.9 . Even with the smaller occurrence rates measured at $10 \mathrm{~s}$ duration, some optimal correlation values exceed 0.9 . The question of what optimal cross-correlation value corresponds to a significantly unchanged scintillation fade pattern is complex. From a practical viewpoint it is related to receiver technology and implementation. Once a receiver loses tracking lock, the change in fade amplitude required to recover is a function of the receiver design, the mean signal amplitude, and the antenna coupling to the receiver. Receivers designed with the understanding that the fade duration will be substantially longer for moving receivers than for stationary receivers will perform better in a scintillating environment.

\section{Conclusions}

[53] GPS L1 C/A code scintillations data were collected over a three-month period from January through March in 2001, yielding about 45 nights of data for five spaced receivers. By analyzing autocorrelation and cross-correlation functions of $40 \mathrm{~s}$ length, the data set was reduced to a set of parameters representing the correlation functions. From these parameters we conclude that the scintillation fade patterns' apparent and true velocities are equal to a good approximation, that eastward (westward) velocities are about $90 \%(10 \%)$ of the total, and that the most likely range of velocities is $100-200 \mathrm{~m} / \mathrm{s}$ eastward.

[54] We also concluded that a simple projection model of fades organized by the magnetic field can explain the shape and orientation of the scintillation fade patterns. The projection model related the measured and calculated projection angles for all of the data but the relationship was only one to one for signal paths separated by at least $60^{\circ}$ from the magnetic field. Several possibilities exist for explaining the anomalous data from signal paths of $60^{\circ}$ or closer to the magnetic field but we could not conclude that any of them were valid. Currently, we do know that many of the signal paths aligned $60^{\circ}$ or closer to the magnetic field yield orientations that agree with the projection model predictions. Using a discriminator of $20 \%$, all of the results were evenly divided between agreement and disagreement with the projection model. This subject will need more analysis before being resolved. The significance of the $60^{\circ}$ boundary is emphasized in our last figure in which Figure 3 is revisited. Experiment validity was tested in Figure 3 by examining drift velocities measured by the U-V and $\mathrm{V}-\mathrm{W}$ receiver pairs compared to measurements by the $\mathrm{U}-\mathrm{W}$ receiver pair. The comparisons should have been lines of slope 1 and intercept 0 but the result was not conclusive. In Figure 15 we make the comparisons again but eliminate events where the signal path is within $60^{\circ}$ of the magnetic field. The comparison is much more satisfying this time and the best case yields a line of slope 0.994 and an intercept of $1.5 \mathrm{~m} / \mathrm{s}$. Clearly, signal paths close to the magnetic field propagate differently than signal paths farther from the magnetic field.

[55] The concept of a projection angle was then applied to understanding the shape and width of the scintillation fade pattern, resulting in scintillation fade patterns that were greatly elongated compared to $1 \mathrm{~km}$ and most likely with Fresnel lengths of about $450 \mathrm{~m}$. The actual elongation was not measurable since the mean optimal cross-correlation amplitude was 0.962 , which is indistinguishable from 1. The width of $450 \mathrm{~m}$ was consistent with weak scattering theory [Salpeter, 1967] that implies a single organizing scale length.

[56] Finally, we examined the time duration of the scintillation fade patterns for all signal paths in the true velocity frame, noting that, to a good approximation, the true and apparent velocities are equal. For $5 \mathrm{~s}$ duration, nearly $50 \%$ of the optimal cross-correlation amplitudes exceeded 0.8 . At $10 \mathrm{~s}$ the data density had dropped to small values but there were still some optimal cross-correlation values exceeding 0.9. Overall we think that the conclusions reached in Kintner et al. [2001] are validated by this data set. Airborne receivers are likely to resonate or match velocities with scintillation fade patterns. The good news is that the distribution of orientations will reduce the number of satellites in resonance for some cases. If the receiver motion is east-west, no advantage exists. 

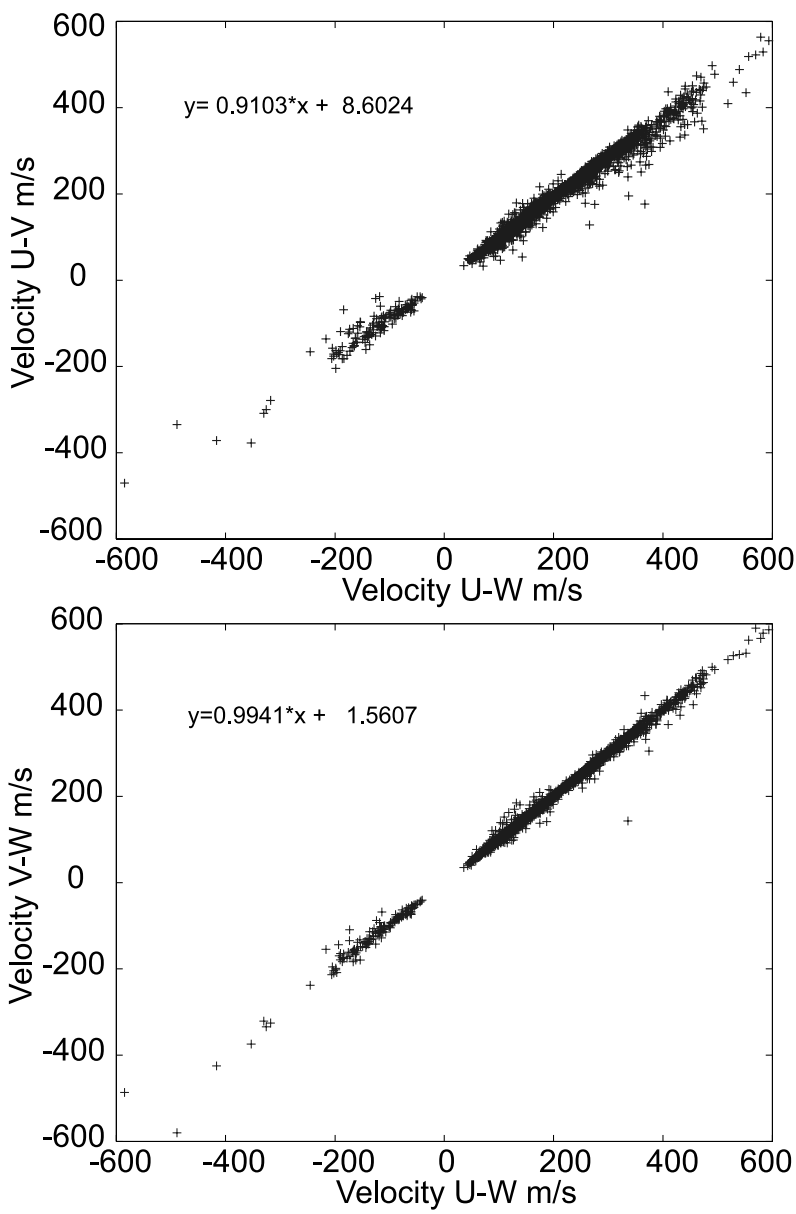

Figure 15. A comparison of scintillation fade pattern true velocities measured using the $\mathrm{U}-\mathrm{V}$ and $\mathrm{V}-\mathrm{W}$ receivers pairs compared to the $\mathrm{U}-\mathrm{W}$ receiver pair measurements, similar to Figure 3. In this case only data with signal paths separated by at least $60^{\circ}$ from the magnetic field direction are used. The parameters from a least squares fit of a straight line are shown in each panel. The comparison in the lower panel is confirmed to at least two decimal places and validates the experiment.

However, if there is a north-south component of the receiver motion, the receiver will resonate only for specific orientations and projection angles.

\section{Appendix A: Measurement of the Projection Angle}

[57] The orientation of a fade surface with respect to magnetic, or geographic, coordinates can be derived from a grid of receivers measuring the relative arrival time of the fade surface at the various receivers. In this example the projection angle of a fade surface using three receivers is calculated. The assumptions are that the fade surface is one-dimensional or at least greatly elongated compared to the distance between the receivers and that the three receivers are not collinear. The velocity of the fade surface in the horizontal plane is arbitrary. Figure A1 shows this arrangement where the three receivers are labeled $\mathrm{a}, \mathrm{b}$, and $\mathrm{c}$, the velocity vector is labeled $\mathbf{V}$, and the normal to the fade surface is labeled k. The projection angle $\phi$ is as defined in section 5. In addition, the assumption of equality between apparent and true velocities is justified in section 4.

[58] In analogy to a propagating wave the fade surface can be thought of as a surface of constant phase where $\mathbf{k} \cdot \mathbf{r}-\omega t=0$. The phase of the propagating wave only depends on the component of $V$ perpendicular to the fade surface, which is $\hat{\mathbf{k}} \cdot \mathbf{V}$. So the equation of a surface of constant phase becomes $|\mathbf{k}|(\hat{\mathbf{k}} \cdot \mathbf{r}-\hat{\mathbf{k}} \cdot \mathbf{V} t)=0$ where $\omega=\hat{\mathbf{k}} \cdot \mathbf{V} /|\mathbf{k}|$. To evaluate the surface of constant phase at the different receivers, the difference vectors $(\mathbf{b}-\mathbf{a})=$ $b \hat{\mathbf{b}}$ and $(\mathbf{c}-\mathbf{a})=c \hat{\mathbf{c}}$ are defined where $\mathbf{a}, \mathbf{b}$, and $\mathbf{c}$ describe the locations of the three receivers $a, b$, and $c$, and where $\hat{\mathbf{b}}$ and $\hat{\mathbf{c}}$ are the unit vectors from the a receiver to the $\mathrm{b}$ and $\mathrm{c}$ receivers, respectively. Finally, the times for the surface to move from receiver a to receivers $b$ and $\mathrm{c}$ are defined as $t_{b}$ and $t_{c}$, respectively.

[59] The surface of constant phase initially measured at receiver $\mathrm{a}$ is then measured at receivers $\mathrm{b}$ and $\mathrm{c}$ as

$$
\hat{\mathbf{k}} \cdot b \hat{\mathbf{b}}-\hat{\mathbf{b}} \cdot \mathbf{V} t_{b}=0
$$

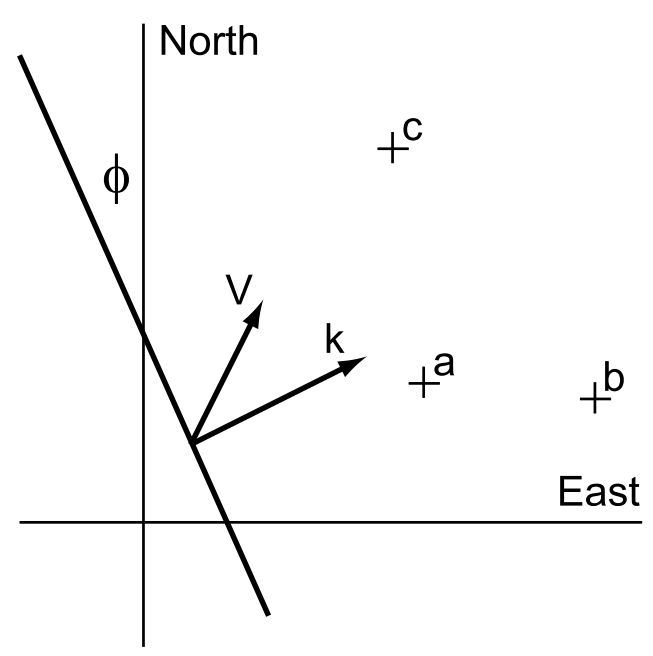

Figure A1. Schematic of the relation between a fade surface with projection angle $\phi$ moving at an arbitrary velocity $\mathbf{V}$. The locations of three receivers are labeled as $\mathrm{a}, \mathrm{b}$, and $\mathrm{c}$. The vector $\mathbf{k}$ is the normal to the fade surface. 
and

$$
\hat{\mathbf{k}} \cdot c \hat{\mathbf{c}}-\hat{\mathbf{k}} \cdot \mathbf{V} t_{c}=0,
$$

which become, when combined,

$$
\frac{\hat{\mathbf{k}} \cdot \hat{\mathbf{b}}}{\hat{\mathbf{k}} \cdot \hat{\mathbf{c}}}=\frac{c}{b} \frac{t_{b}}{t_{c}} .
$$

If $\hat{\mathbf{b}}$ and $\hat{\mathbf{c}}$ are in the northward and eastward directions, respectively, this relation can be written in terms of the projection angle $\phi$ as

$$
\frac{\sin (\phi)}{\cos (\phi)}=\frac{c}{b} \frac{t_{b}}{t_{c}} .
$$

If $\hat{\mathbf{b}}$ and $\hat{\mathbf{c}}$ are not in the northward and eastward directions, respectively, the corrections are trivial. Surprisingly, the relation between the projection angle $\phi$ and the lag times $t_{b}$ and $t_{c}$ is completely independent of the fade surface velocity vector direction and magnitude. We also note that our approach could be more rigorous by describing the fade surface with a Fourier decomposition.

[60] Acknowledgments. Research at Cornell University was funded by the Office of Naval Research under grant N00014-92-J-1822. The authors thank INPE for their support and assistance, with special thanks to engineers Sinval Domingos and Fabiano S. Rodrigues and to technicians Patrícia M. de Siqueira and Cristiano de Castilho for the GPS installation, operation, and data processing at Cachoeira Paulista. One of us, E. R. de Paula, would like to thank CNPq under process 502223/91-0 and FAPESP under process 97/3342-5 for the partial support. The initial reduction of data to correlation parameters was performed by Cornell undergraduate Lars Kalnajs.

\section{References}

Aarons, J. (1982), Global morphology of ionospheric scintillations, Proc. IEEE, 70(4), 360-378.

Aarons, J. (1993), The longitudinal morphology of equatorial $F$-layer irregularities relevant to their occurrence, Space Sci. Rev., 63(3-4), 209-243.

Aarons, J. (1997a), 50 years of radio-scintillation observations, IEEE Antennas Propag. Mag., 39(6), 7-12.

Aarons, J. (1997b), Global Positioning System phase fluctuations at auroral latitudes, J. Geophys. Res., 102(8), 17,21917,232 .

Aarons, J., J. P. Mullen, H. E. Whitney, and E. M. MacKenzie (1980), The dynamics of equatorial irregularity patch formation, motion, and decay, J. Geophys. Res., 85(1), 139-149.

Aarons, J., J. A. Klobuchar, H. E. Whitney, J. Austen, A. L. Johnson, and C. L. Rino (1983), Gigahertz scintillations associated with equatorial patches, Radio Sci., 18(3), $421-434$.
Aarons, J., M. Mendillo, and R. Yantosca (1996), GPS phase fluctuations in the equatorial region, paper presented at Ionospheric Effects Symposium, Alexandria, Va.

Aarons, J., M. Mendillo, and R. Yantosca (1997), GPS phase fluctuations in the equatorial region during sunspot minimum, Radio Sci., 32(4), 1535-1550.

Abdu, M. A., I. J. Kantor, I. S. Batista, and E. R. de Paula (1985a), East-west bubble irregularity motion determined from spaced VHF polarimeters: Implications on velocity shear in the zonal $F$ region bulk plasma motion, Radio Sci., 20(1), 111-122.

Abdu, M. A., I. S. Batista, J. H. A. Sobral, E. R. de Paula, and I. J. Kantor (1985b), Equatorial ionospheric plasma bubble irregularity occurrence and zonal velocities under quiet and disturbed conditions from polarimeter observations, J. Geophys. Res., 90(10), 9921-9928.

Abdu, M. A., J. H. A. Sobral, Y. Nakamura, and C. J. Zamlutti (1987), Equatorial plasma bubble zonal velocity height gradient from spaced VHF polarimeter and scanning 630-nm measurements, Geophys. Res. Lett., 14(9), 965-968.

Abdu, M. A., J. H. A. Sobral, I. S. Batista, V. H. Rios, and C. Medina (1998), Equatorial spread $F$ occurrence statistics in the American longitudes: Diurnal, seasonal and solar cycle variations, $A d v$. Space Res., 22(6), 851-854.

Aggson, T. L., N. C. Maynard, F. A. Herrero, H. G. Mayr, L. H. Brace, and M. C. Liebrecht (1987), Geomagnetic equatorial anomaly in zonal plasma flow, J. Geophys. Res., 92(1), $311-315$.

Basu, S., and S. Basu (1981), Equatorial scintillations-A review, J. Atmos. Terr. Phys., 43(5-6), 473-489.

Basu, S., J. Aarons, J. P. McClure, C. La Hoz, A. Bushby, and R. F. Woodman (1977), Preliminary comparisons of VHF radar maps of $F$ region irregularities with scintillations in the equatorial region, J. Atmos. Terr. Phys., 39(9-10), 12511261.

Basu, S., J. P. McClure, S. Basu, W. B. Hanson, and J. Aarons (1980), Coordinated study of equatorial scintillations and in situ and radar observations of nighttime $F$ region irregularities, J. Geophys. Res., 85(10), 5119-5130.

Basu, S., S. Basu, J. LaBelle, E. Kudeki, B. G. Fejer, M. C. Kelley, H. E. Whitney, and A. Bushby (1986), Gigahertz scintillations and spaced receiver drift measurements during Project Condor equatorial $F$ region rocket campaign in Peru, J. Geophys. Res., 91(5), 5526-5538.

Basu, S., S. Basu, E. Kudeki, H. P. Zengingonul, M. A. Bioni, and J. W. Meriwether (1991), Zonal irregularity drifts and neutral winds measured near the magnetic equator in Peru, J. Atmos. Terr. Phys., 53(8), 743-755.

Basu, S., et al. (1996), Scintillations, plasma drifts, and neutral winds in the equatorial ionosphere after sunset, J. Geophys. Res., 101(12), 26,795-26,809.

Beach, T. L., and P. M. Kintner (1999), Simultaneous Global Position System observations of equatorial scintillations and total electron content fluctuations, J. Geophys. Res., 104(10), 22,553-22,565. 
Beach, T. L., and P. M. Kintner (2001), Development and use of a GPS ionospheric scintillation monitor, IEEE Trans. Geosci. Remote Sens., 39(5), 918-928.

Bhattacharyya, A., S. Basu, K. M. Groves, C. E. Valladares, and R. Sheehan (2001), Dynamics of equatorial $F$ region irregularities from spaced receiver scintillation observations, Geophys. Res. Lett., 28(1), 119-122.

Bishop, G., S. Basu, E. Holland, and J. Secan (1994), Impacts of ionospheric fading on GPS navigation integrity, in Proceedings of ION GPS-94, pp. 577-585, Inst. of Navig., Fairfax, Va.

Briggs, B. H., G. J. Phillips, and D. H. Shinn (1950), The analysis of observations on spaced receivers of the fading of radio signals, Proc. Phys. Soc., Sect. B, 63, 106-121.

Coley, W. R., and R. A. Heelis (1989), Low-latitude zonal and vertical ion drifts seen by DE 2, J. Geophys. Res., 94(6), $6751-6761$.

DasGupta, A., S. Basu, J. Aarons, J. A. Klobuchar, S. Basu, and A. Bushby (1983), VHF amplitude scintillations and associated electron content depletions as observed at Arequipa, Peru, J. Atmos. Terr. Phys., 45(1), 15-26.

Doherty, P. H., S. H. Delay, C. E. Valladares, and J. Klobuchar (2000), Ionospheric scintillation effects in the equatorial and auroral regions, in Proceedings of ION GPS-2000, pp. $662-$ 671, Inst. of Navig., Fairfax, Va.

Farley, D. T., B. B. Balsley, R. F. Woodman, and J. P. McClure (1970), Equatorial spread $F$ : Implications of VHF radar observations, J. Geophys. Res., 75(34), 7199-7216.

Fejer, B. G., D. T. Farley, C. A. Gonzales, and R. F. Woodman (1981), $F$ region east-west drifts at Jicamarca, J. Geophys. Res., 86(1), 215-218.

Fejer, B. G., L. Scherliess, and E. R. de Paula (1999), Effects of the vertical plasma drift velocity on the generation and evolution of equatorial spread F, J. Geophys. Res., 104(9), 19,859-19,869.

Goodman, J. L., and L. Kramer (2001), Scintillation effects on space shuttle GPS data, paper presented at National Technical Meeting, Inst. of Navig., Long Beach, Calif., 22-24 Jan.

Hofmann-Wellenhof, B., H. Lichtenegger, and J. Collins (2001), GPS Theory and Practice, 5th ed., Springer-Verlag, New York.

Hysell, D. L. (1999), Imaging coherent backscatter radar studies of equatorial spread F, J. Atmos. Sol. Terr. Phys., 61(9), $701-716$.

Kelley, M. C., G. Haerendel, H. Kappler, A. Valenzuela, B. B. Balsley, D. A. Carter, W. L. Ecklund, C. W. Carlson, B. Hausler, and R. Torbert (1976), Evidence for a Rayleigh-Taylor type instability and upwelling of depleted density regions during equatorial spread $F$, Geophys. Res. Lett., 3(8), 448-450.

Kelley, M. C., D. Kotsikopoulos, T. Beach, D. Hysell, and S. Musman (1996), Simultaneous Global Positioning System and radar observations of equatorial spread $F$ at Kwajalein, J. Geophys. Res., 101(2), 2333-2341.
Kil, H., P. M. Kintner, E. R. de Paula, and I. J. Kantor (2000), Global Positioning System measurements of the ionospheric zonal apparent velocity at Cachoeira Paulista in Brazil, J. Geophys. Res., 105(3), 5317-5327.

Kil, H., P. M. Kintner, E. R. de Paula, and I. J. Kantor (2002), Latitudinal variations of scintillation activity and zonal plasma drifts in South America, Radio Sci., 37(1), 1006, doi:10.1029/2001RS002468.

Kintner, P. M., H. Kil, T. L. Beach, and E. R. de Paula (2001), Fading timescales associated with GPS signals and potential consequences, Radio Sci., 36(4), 731-743.

Kramer, L., and J. L. Goodman (2001), Global scale observations of ionospheric instabilities from GPS in low earth orbit, paper presented at Space 2001...The Odyssey Continues Conference and Exposition, Am. Inst. of Aeron. and Astron., Albuquerque, N. M., 28-30 Aug.

McClure, J. P., W. B. Hanson, and J. H. Hoffman (1977), Plasma bubbles and irregularities in the equatorial ionosphere, J. Geophys. Res., 82(19), 2650-2656.

Mendillo, M., and J. Baumgardner (1982), Airglow characteristics of equatorial plasma depletions, J. Geophys. Res., 87(9), 7641-7652.

Mendillo, M., J. Baumgardner, M. Colerico, and D. Nottingham (1997), Imaging science contributions to equatorial aeronomy: Initial results from the MISETA program, J. Atmos. Sol. Terr. Phys., 59(13), 1587-1599.

Musman, S., J.-M. Jahn, J. LaBelle, and W. E. Swartz (1997), Imaging spread $F$ structures using GPS observations at Alcântara, Brazil, Geophys. Res. Lett., 24(13), 1703-1706.

Pi, X., A. J. Mannucci, U. J. Lindqwister, and C. M. Ho (1997), Monitoring of global ionospheric irregularities using the worldwide GPS network, Geophys. Res. Lett., 24(18), 2283-2286.

Rickett, B. (2001), Radio sources and scintillation, Astrophys. Space Sci., 278(1-2), 5-10.

Rino, C. L., and J. Owen (1980), The time structure of transionospheric radio wave scintillation, Radio Sci., 15(3), 479-489.

Salpeter, E. E. (1967), Interplanetary scintillations, I, Theory, Astrophys. J., 147(2), 433-438.

Sobral, J. H. A., and M. A. Abdu (1991), Solar activity effects on equatorial plasma bubble zonal velocity and its latitude gradient as measured by airglow scanning photometers, J. Atmos. Terr. Phys., 53(8), 729-742.

Sobral, J. H. A., M. A. Abdu, S. Yamashita, W. D. Gonzalez, A. C. de Gonzalez, I. S. Batista, C. J. Zamlutti, and B. T. Tsurutani (2001), Responses of the low-latitude ionosphere to very intense geomagnetic storms, J. Atmos. Sol. Terr. Phys., 63(9), 965-974.

Spatz, D. E., S. J. Franke, and K. C. Yeh (1988), Analysis and interpretation of spaced receiver scintillation data recorded at an equatorial station, Radio Sci., 23(3), 347-361.

Tinsley, B. A., R. P. Rohrbaugh, W. B. Hanson, and A. L. Broadfoot (1997), Images of transequatorial $F$ region bubbles in 630- and 777-nm emissions compared with satellite measurements, J. Geophys. Res., 102(2), 2057-2077. 
Tokumaru, M., M. Kojima, K. Fujiki, and A. Yokobe (2000), Three-dimensional propagation of interplanetary disturbances detected with radio scintillation measurements at $327 \mathrm{MHz}$, J. Geophys. Res., 105(5), 10,43510,453 .

Vacchione, J. D., S. J. Franke, and K. C. Yeh (1987), A new analysis technique for estimating zonal irregularity drifts and variability in the equatorial $F$ region using spaced receiver scintillation data, Radio Sci., 22(5), 745756.

Valladares, C. E., R. Sheehan, S. Basu, H. Kuenzler, and J. Espinoza (1996), The multi-instrumented studies of equatorial thermosphere aeronomy scintillation system: Climatology of zonal drifts, J. Geophys. Res., 101(12), 26,83926,850 .

Vats, H. O., S. Sharma, R. Oza, K. N. Iyer, H. Chandra, H. S. Sawant, and M. R. Deshpande (2001), Interplanetary and terrestrial observations of an Earth-directed coronal mass ejection, Radio Sci., 36(6), 1769-1773.
Woodman, R. F. (1972), East-west ionospheric drifts at the magnetic equator, Space Res., 12, 969-974.

Woodman, R. F., and C. La Hoz (1976), Radar observations of $F$ region irregularities, J. Geophys. Res., 81(31), 54475466.

Yeh, K. C., and C. H. Liu (1982), Radio wave scintillations in the ionosphere, Proc. IEEE, 70(4), 324-360.

Yeh, K. C., C. H. Liu, and M. Y. Youakim (1975), A theoretical study of the ionospheric scintillation behavior caused by multiple scattering, Radio Sci., 10(1), 97-106.

E. R. de Paula and I. J. Kantor, Aeronomy Division, DAE/ CEA, INPE, São José dos Campos, 12201-970 São Paulo, Brazil.

P. M. Kintner and B. M. Ledvina, School of Electrical and Computer Engineering, Cornell University, Ithaca, NY 148533801, USA.(paul@ece.cornell.edu) 


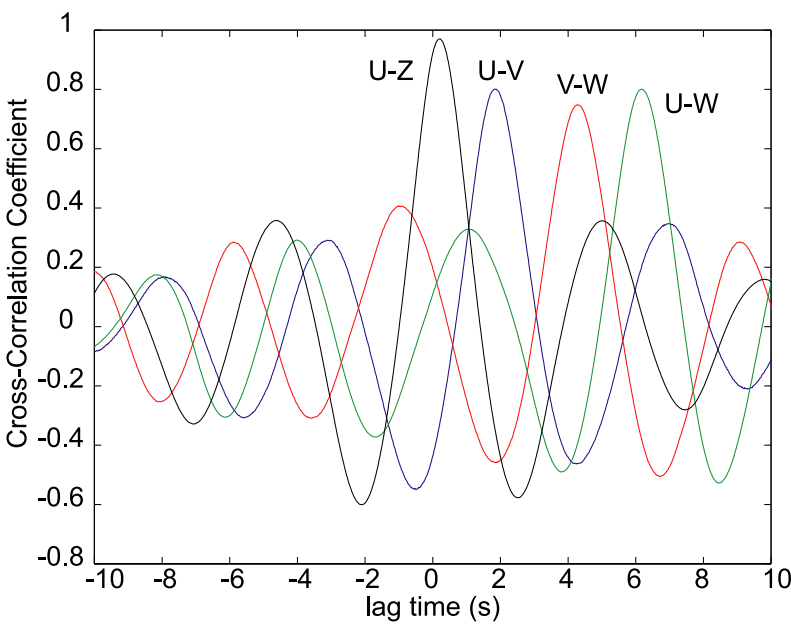

Figure 2. Examples of normalized cross-correlation functions between different receiver pairs for a single GPS satellite signal. Note that the receiver pair aligned north-south (U-Z) has larger optimal correlation values and smaller times of optimal correlation compared to the receivers in the east-west direction $(\mathrm{U}-\mathrm{V}, \mathrm{V}-\mathrm{W}$, and $\mathrm{U}-\mathrm{W})$.
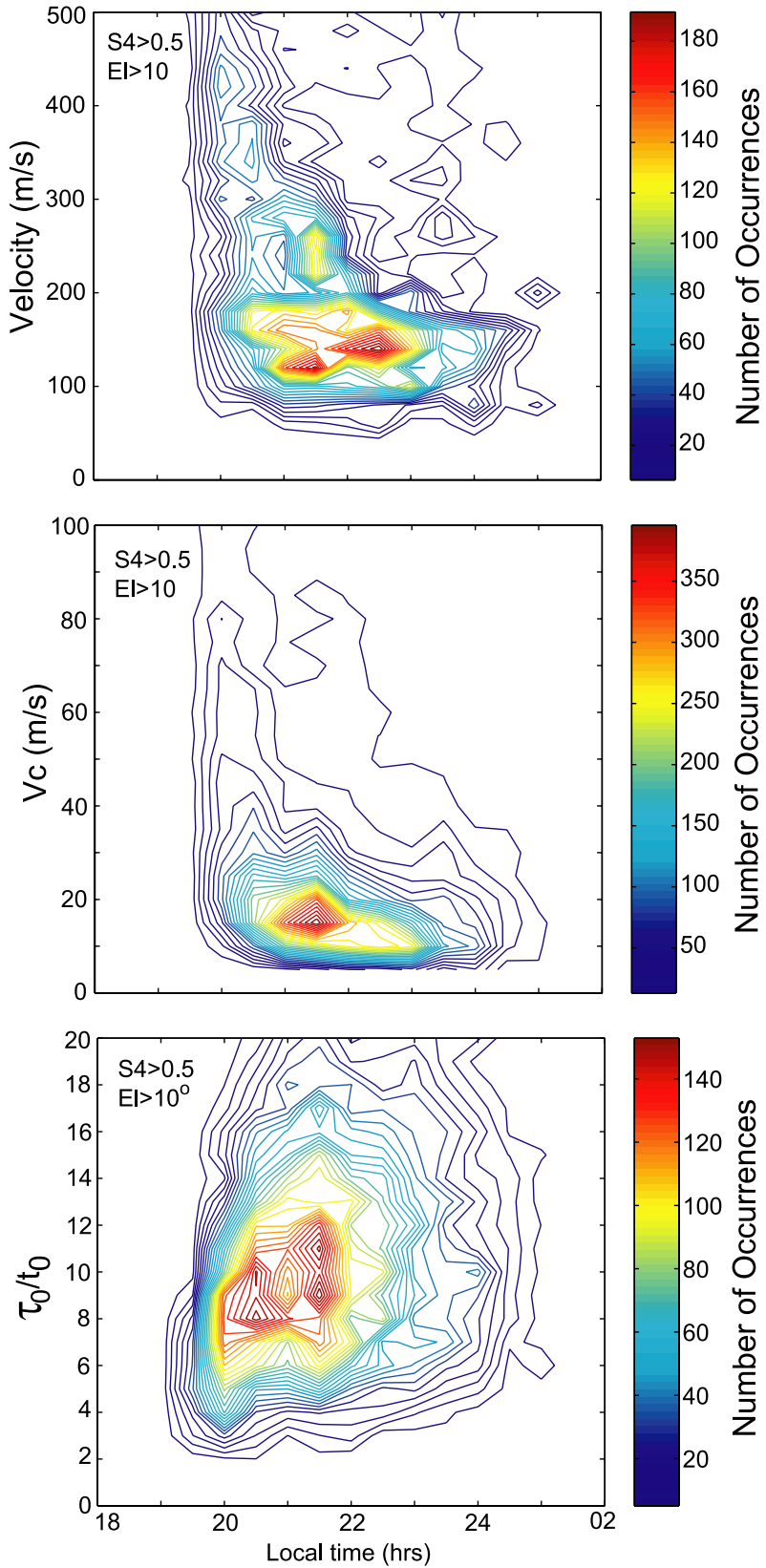

Figure 5. Contour plots of occurrence rates for the true eastward velocity (top), the characteristic velocity (middle), and the ratio $\tau_{0} / t_{0}$ (bottom) versus local time. Shown are the number of occurrences of true velocity in bins of $0.5 \mathrm{~h}$ by $25 \mathrm{~m} / \mathrm{s}$ (top), the number of occurrences of characteristic velocity in bins of $0.5 \mathrm{~h}$ by $5 \mathrm{~m} / \mathrm{s}$ (middle), and the number of occurrences of the ratio $\tau_{0} / t_{0}$ (bottom) in a bin of $0.5 \mathrm{~h}$ by 1 of the ratio. 


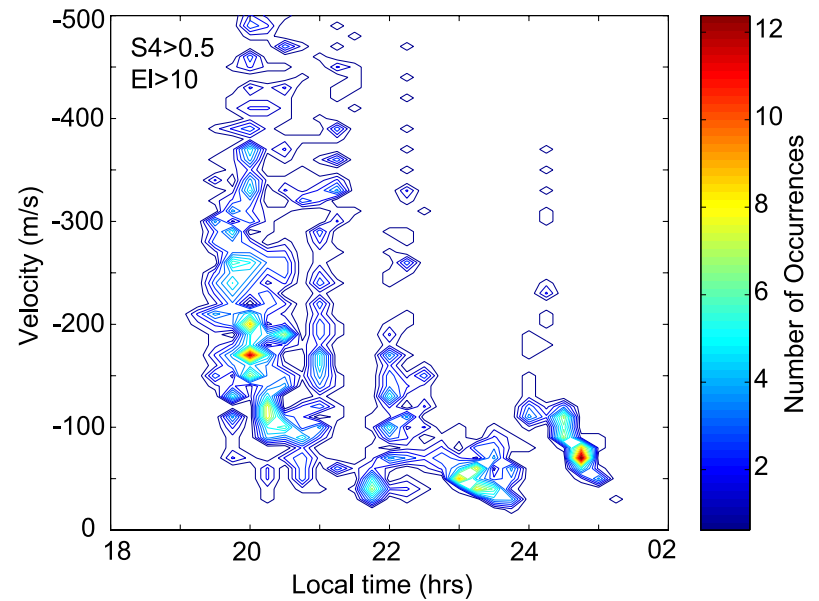

Figure 6. Contour plots of the number of occurrences of negative (westward) true velocity in bins of $0.25 \mathrm{~h}$ by $25 \mathrm{~m} / \mathrm{s}$ versus local time.

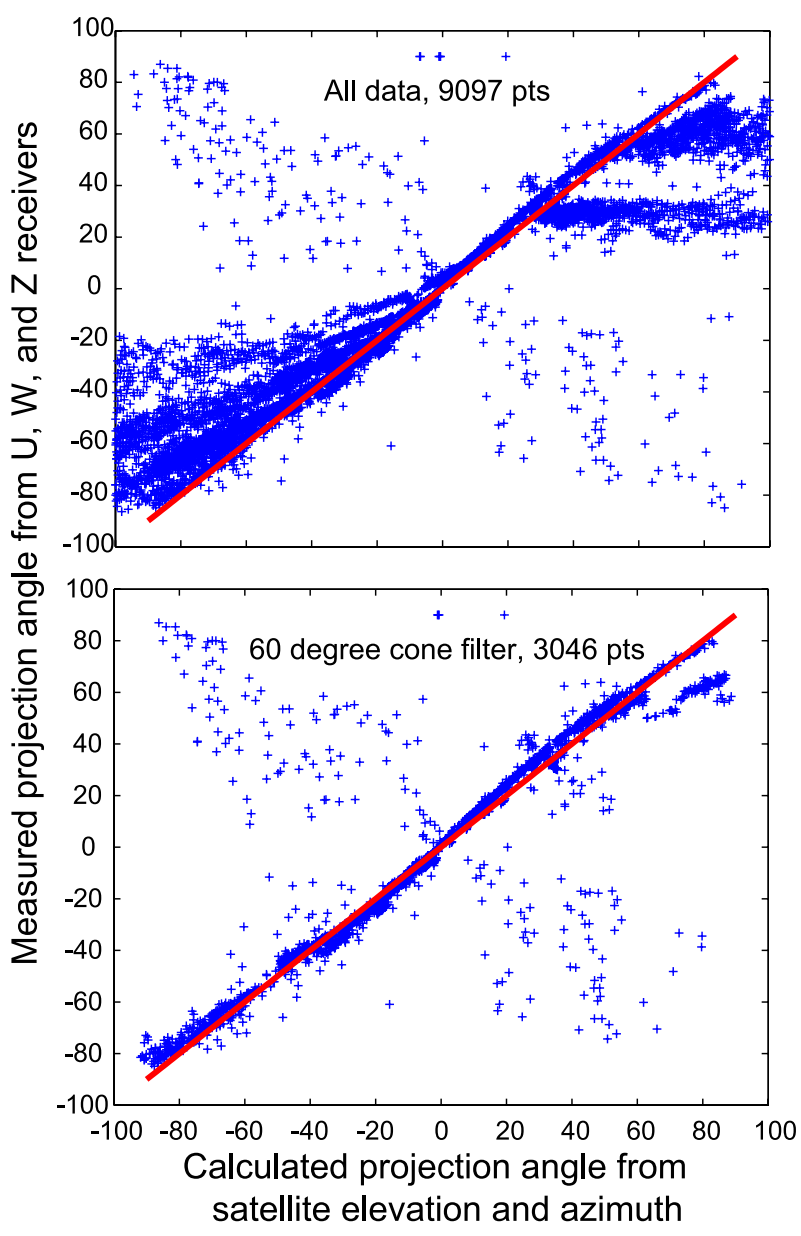

Figure 9. A comparison of calculated and measured projection angles. (top) All available data are shown. (bottom) Only data for signal paths separated by at least $60^{\circ}$ from the magnetic field direction are shown. The red line is the expected result for the projection model. 

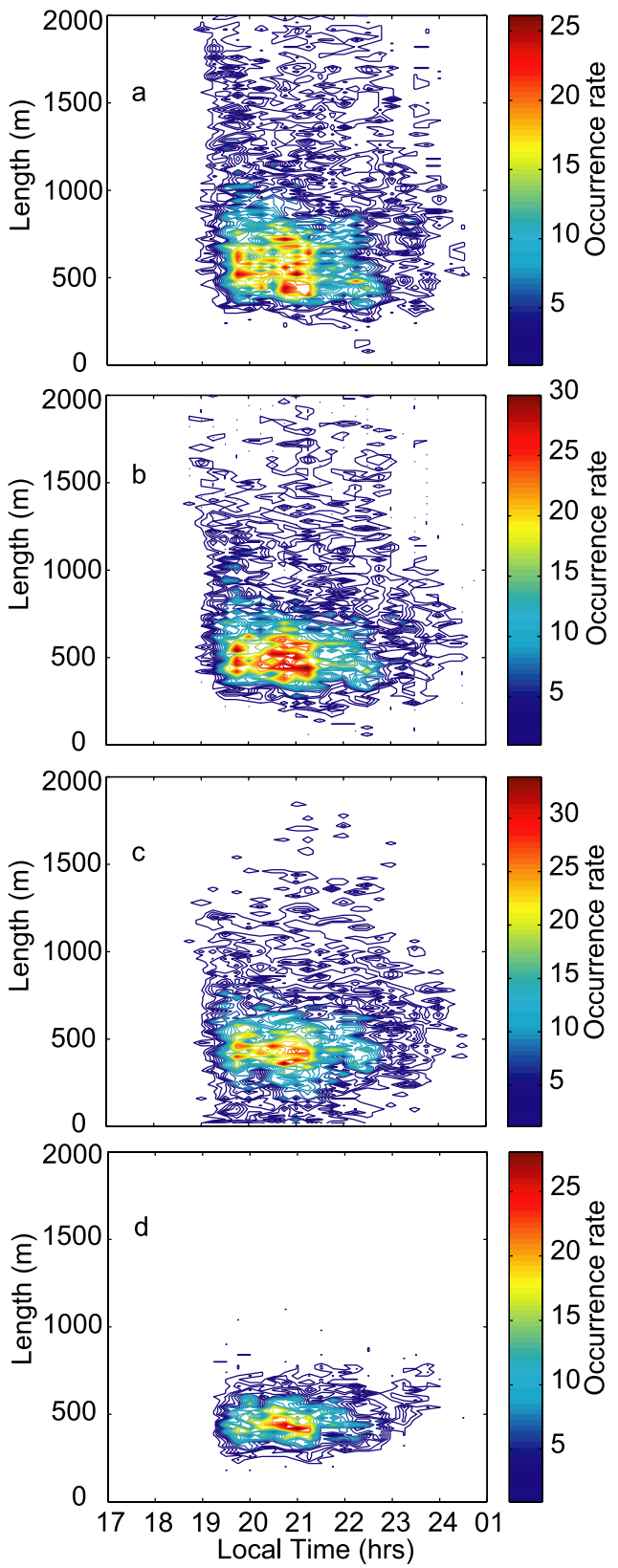

Figure 11. Contour plots of occurrence rates for different methods of estimating the measured Fresnel length. (a) Makes no adjustments. (b) Adjusts for slant range only. (c) Adjusts for slant range and projection angle. (d) Adjusts for slant range and projection angle and only uses data whose signal paths are separated by at least $60^{\circ}$ from the magnetic field direction.

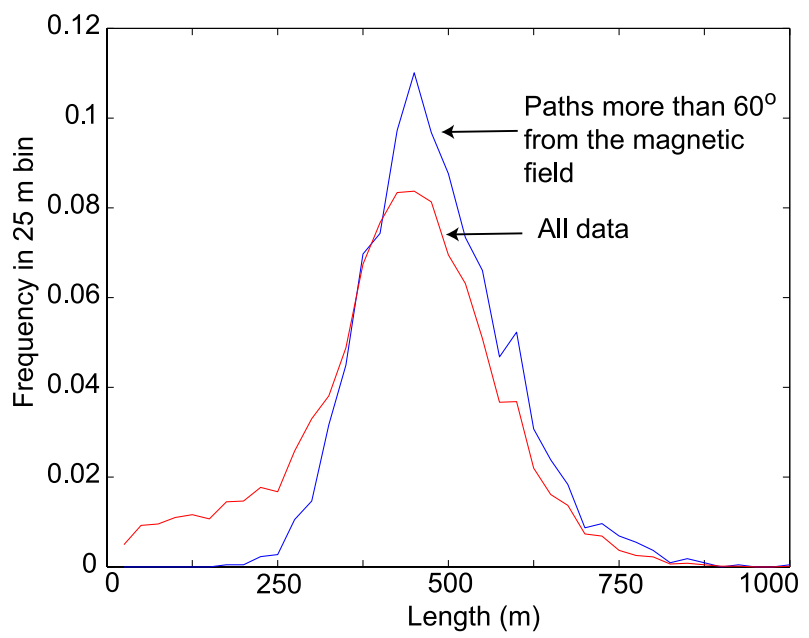

Figure 12. Probability distributions for the measured fade width within $25 \mathrm{~m}$ bins. Shown are the distributions reduced from Figures $11 \mathrm{c}$ and $11 \mathrm{~d}$. The red curve labeled "all data" corresponds to Figure 11c where the fade widths have been adjusted for slant range and orientation. The blue curve labeled "paths more than $60^{\circ}$ from the magnetic field line" corresponds to Figure $11 \mathrm{~d}$ and includes all data adjusted for slant range and orientation with signal paths aligned at least $60^{\circ}$ from the magnetic field direction.

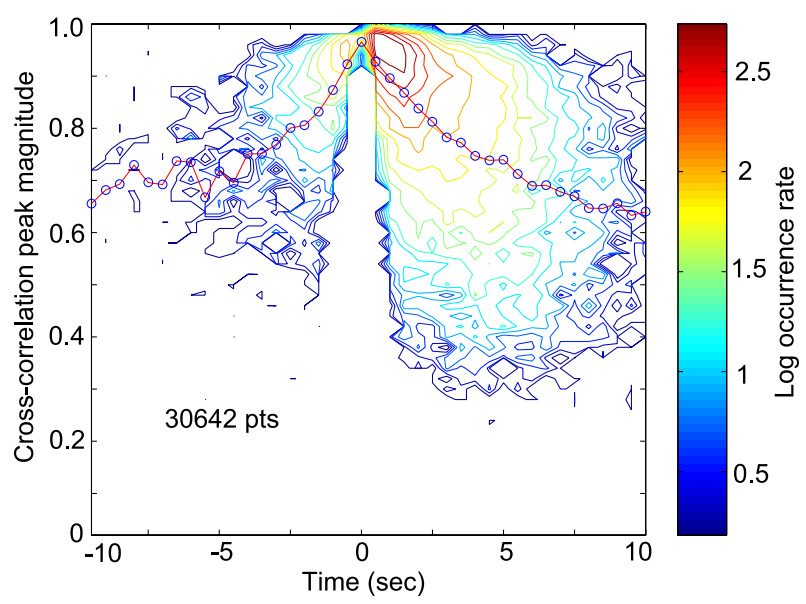

Figure 13. Evolution of the optimal cross-correlation amplitude as a function of time. The logarithm of the occurrence rate in bins of $0.5 \mathrm{~s}$ by 0.02 correlation amplitude are shown on a contour plot. Positive time represents eastward flows and negative time represents westward flows. The mean in each $0.5 \mathrm{~s}$ bin is also plotted. 